BABELAO 2 (2013), p. 3-52

(C) ABELAO (Belgium)

\title{
Eléments de morphologie et de syntaxe de la langue hourrite Destinés à l'étude des textes mittaniens et anatolo-hittites
}

Par

\author{
Arnaud Fournet \\ La Garenne Colombes, France
}

I 'article a pour objectif central de décrire la morphologie, les classes lexicales et la syntaxe de la langue hourrite, dans ce qu'elle a de mieux comprise, avec l'optique particulière de faciliter l'étude philologique des documents en langue hourrite d'origine mittannienne et anatolo-hittite. Le point de référence pris en compte pour la description est l'état de langue reflété dans la Lettre du Mittanni, un document assurément écrit par des locuteurs hourrites. La langue décrite ci-dessous est dans une certaine mesure normalisée, et elle gomme la variation observée dans les textes, tant dans les graphies que les formes. Elle sera appelée hourrite classique normalisé $(\mathrm{HCN})$. Cette opération a pour but de fournir une référence universelle, aussi naturelle et fidèle que possible, avec laquelle on puisse élucider les formes réellement attestées, soit directement soit par écart. Ces écarts, dont l'origine tient principalement au grand laps de temps qui sépare les différents documents, et peut-être à des variantes dialectales, sont également décrits. 
Les questions comparatives, étymologiques, historiques, phonétiques et graphémiques sont réduites au strict minimum et ne sont évoquées que dans la limite de leur pertinence par rapport à l'objectif de décrire une sorte de hourrite normalisé et les écarts pratiques à cette norme. La partie théorique (\$.1 à 14) est suivie d'un exemple de mise en oeuvre des principes grammaticaux.

\section{Introduction à la problématique hourrite}

1.1. Le hourrite est une langue, désormais éteinte, qui fut parlée en Anatolie et dans le nord de la Mésopotamie. Elle est attestée sous forme écrite pendant environ mille ans à partir du dernier tiers du $3^{\text {ème }}$ millénaire jusqu'au milieu du $13^{\text {ème }}$ siècle avant J.C. Dans les textes francophones du début du $20^{\text {ème }}$ siècle on trouve la variante orthographique hurrite, dans laquelle le digraphe $<$ ou $>$ est noté avec la seule lettre $<\mathrm{u}>$. Cette langue était appelée burlili en hittite, $\langle>>$ [ r g $]$ en alphabet ougaritique, ce qui se vocalise $* /$ xurru $\gamma$ i/ en alphabet phonétique international ou *burrugi si l'on adopte les conventions sémitologiques. Le terme bourrite lui-même, et ses variantes contemporaines : anglais hurrian, allemand burritisch, italien burrico, etc., pour la langue, et anglais Hurrian, allemand Hurriter, italien Hurrito, etc., pour les locuteurs hourrites, repose sur l'auto-ethnonyme burrubi, et sa variante $<$ hur-wu-hi $>$, d'apparence phonétique archaïsante $<$ rw $\rangle$, où l'on reconnaît le suffixe - bi- grâce auquel le hourrite forme des ethnonymes. La base hurri est par exemple attestée dans $<$ Hər-ri a-ma-

$t^{\mathrm{MEŠ}}>$ 'les ancêtres hourrites' (KBo I 3 Vo 27) figurant dans un traité conclu entre Hourrites et Hittites. En langue hattie, parlée au nord-ouest des Hourrites, et qui est le substrat de la langue hittite, aussi appelée nésite, l'ethnonyme des Hourrites se dit <wahər-li $>$, dans lequel $<$ wa- $>$ exprime en hatti le pluriel de la base lexicale $<$ hər-li $>$.

1.2. L'auto-ethnonyme burrubi est écrit $<$ hər-ru-hi $>$ dans la Lettre du Mittanni avec un signe cunéiforme à lecture multiple : hur ou har. Laroche ${ }^{1}$ semble émettre un doute quant à la voyelle radicale de hurrubi et hurwubi. Pourtant, quelque cinquante ans plus tôt, Ungnad ${ }^{2}$ avait fait remarquer que ce signe, certes a priori ambigu, semble dans les faits toujours décomposé bu-ur et jamais **ba-ar, confirmant ainsi la voyelle [u]. Un point d'historiographie hourritologique est fourni par Gelb ${ }^{3}$ : «the name of the [Hurrian]

1 E. Laroche, Glossaire de la langue hourrite (= Revue Hittite et Asianique, 34/35), Paris, 1980, p. 14.

2 A. UNGNAD, «Hurriland und Mitanni », Zeitschrift für Assyriologie und Vorderasiatische Archäologie 36 (1925), p. 101.

3 I.J. GELB, Hurrians and Subarians. Chicago (Illinois), 1944, p. 50-51. «Le nom du peuple hourrite fut d'abord lu Harri, l'identité supposée de ce nom avec celui des Aryens servant d'appui à cette lecture. Cependant il fut vite mon- 
people was at first read as Harri, the alleged identity of this name with "Aryan" serving as support for the reading. Soon, however, it was shown that the HAR-ri could not be Aryans, because their language was not Indo-European. Consequently the reading Harri was abandoned in favor of Hurri ».

1.3. Le hourrite est surtout connu comme étant la langue de ce qui fut autrefois le royaume du Mittanni ${ }^{4}$. Il est probable que la majeure partie des habitants du Mittanni parlaient hourrite et que cette région est leur foyer d'origine. La période mittannienne, qui va d'environ -1500 à -1300, constitue l'apogée de l'influence et de la présence des Hourrites au Proche-Orient ancien. Au début du $2^{\text {ème }}$ millénaire avant J.C, le hourrite était parlé au sud-est de l'Anatolie et dans la région du Zagros-Taurus au nord de la Mésopotamie. Puis au milieu du $2^{\text {ème }}$ millénaire l'influence hourrite croît et s'étend à la Syrie et à la Cilicie (Kizzuwatna). Après la conquête assyrienne des régions peuplées par les Hourrites, au nord de la Mésopotamie, beaucoup d'entre eux furent déportés par Tukulti-Ninurta I (de -1244 à -1208), qui avait besoin de main d'œuvre pour bâtir sa nouvelle capitale. Ces évènements ont certainement causé des torts considérables à la perpétuation de la langue hourrite. En conséquence probable de ces faits tragiques il n'existe pas d'attestation directe de la langue hourrite au $1^{\text {er }}$ millénaire avant J.C mais Gelb ${ }^{5}$ mentionne plusieurs noms de princes hourrites à cette époque tardive, que les Assyriens durent combattre dans les montagnes du nord(-est) de la Mésopotamie.

1.4. Le hourrite est apparenté à l'ourartéen, une langue attestée au $1^{\text {er }}$ millénaire avant J.C au nord-est du domaine hourrite, dans la zone où l'on découvre plus tard le peuplement arménien. Quelques dizaines de mots arméniens sont reconnaissables comme des survivances lexicales du substrat hourro-ourartéen, qui existait avant que la langue arménienne, de filiation indoeuropéenne et proche du grec, ne fasse intrusion et ne conduise au remplacement de l'ourartéen. Diakonov et Greppin ont beaucoup contribué à inventorier ces mots substratiques en arménien ${ }^{6}$. Ces lexèmes sont intéressants car ils éclairent la phonologie de l'ourartéen, tant pour les voyelles que les consonnes, et aussi de façon indirecte la phonétique implicite des signes cunéiformes. Bien qu'il soit attesté après le hourrite, l'ourartéen ne descend pas de cette langue mais constitue une branche sœur. La parenté du hourrite et de l'ourartéen est admise depuis les années 1930. Elle est mentionnée par Speiser, Gelb, etc. Elle a été décrite et détaillée dans les travaux de Diakonov sur cette question dans les

tré que les HAR-ri ne pouvaient pas être “aryens” car leur langue n'était pas indo-européenne. En conséquence la lecture Harri fut abandonnée en faveur de Hurri».

${ }^{4}$ Les raisons de préférer cette graphie sont expliquées plus loin.

${ }^{5}$ GELB, op. cit. (n. 3), p. 81-83.

${ }^{6}$ Voir en particulier les articles de GREPPIN dans la revue Aramazd. 
années 1950 et 1960. Le kassite est un troisième membre de cette famille ${ }^{7}$.

1.5. La communis opinio des comparatistes ne reconnait pas d'apparentement de plus haut niveau pour cette famille hourroourarto-kassite. L'école comparatiste russe de Moscou promeut une connection avec les langues "caucasiques", à la suite des travaux de Diakonov et Starostin ${ }^{8}$. Un article récent appliquant l'approche moscovite a été publié par Kassian'. La principale objection à cette hypothèse est que le groupe géographique « caucasique » n'est pas reconnu lui-même comme un nœud génétique valide par les spécialistes de ces langues. A mon sens il conviendrait de creuser l'hypothèse d'une connection forte entre la famille hourro-ourarto-kassite et spécifiquement le nakh (čečen, inguš, batsbi). De bons comparanda existent entre le hourrite et le nakh. J'ai également proposé de comparer le hourrite avec l'indoeuropéen en collaboration avec A. Bomhard ${ }^{10}$. Cette hypothèse doit être développée et approfondie. Dans l'état actuel du dossier, il est probable que les indo-européanistes ne sont pas disposés à admettre cette parenté.

1.6. Certains mots hourrites attestent des emprunts qui ne sont pas intermédiés par le sémitique comme le mot hourrite tabiri 'forgeron', dérivé du verbe tab- 'couler, fondre (un métal)' et emprunté en sumérien sous la forme tibira 'orfèvre, travailleur de métal', où le vocalisme $i \_i$ est sans doute une harmonisation du sumérien, qui se retrouve dans d'autres mots. Il n'est pas rare que le hourrite présente des formes plus archaïques que celles qu'on rencontre en Mésopotamie : (1) hai-galli 'palais' se laisse comparer au sumérien é-gal 'maison grande'. Plusieurs langues sémitiques, dont l'hébreu ${ }^{11}$ ou l'ougaritique hkl *hēkallu, reposent également sur un prototype *haykal plus ancien que la forme sumérienne conventionnelle é-gal. En arabe classique on trouve haykalun 'grand édifice, grand temple', de la même origine sumérienne ; (2) zuwadatte 'un quart de kor' < akkadien (SG.) sütu (PL.) sütäte '10 qū (8 ou 9 l)'. Le mot hourrite présente la séquence explicite *[uwa], qui peut être postulée comme prototype morphologique sousjacent mais qui est représentée par une voyelle longue contracte [ū] dans les langues sémitiques historiques. Le hourrite confirme l'existence de la séquence *[uwa] à un stade prélittéraire des langues sémitiques.

\footnotetext{
7 Cf. A. Fournet, «The Kassite Language in a Comparative Perspective with Hurrian and Urartian », The Macro-Comparative Journal 2/1 (2011), p. ?

${ }^{8}$ Voir en particulier I.M. DiAKONOV - S.A. STAROSTIN, Hurro-Urartian as an Eastern Caucasian Language, München, 1986.

9 A. KASSIAN, "Hurro-Urartian from the lexicostatical viewpoint », Ugarit Forschungen 42 (2011), p. 383-450.

${ }^{10}$ Voir A. FOURNET - A.R. BOMHARD, The Indo-European Elements in Hurrian, La Garenne Colombes-Charleston, 2010.

${ }^{11}$ Cf. le mot heykeal par exemple, avec vélaire spirante, dans la Bible hébraïque (1 R 6,3).
} 
1.7. Il n'existe aucune raison de faire venir les Hourrites d'un ailleurs hypothétique à une date plus ou moins récente, bien que divers auteurs mentionnent ou imaginent ce genre de migrations et pérégrinations préhistoriques. Les plus proches parents du hourrite, à savoir l'ourartéen et le kassite, sont attestés au nord-est et au sud-est de la zone où il est attesté à date historique. Tout porte à croire que ces trois langues sont présentes à cet endroit depuis un horizon temporel insondable.

1.8. Le corpus hourrite et mittannien est remarquable par la présence inattendue de lexèmes et d'anthroponymes d'allure indoiranienne archaïque. Un point et un examen critique du corpus mittanni-aryen se trouvent dans Fournet (2012) ${ }^{12}$.

\section{Présentation de la langue hourrite}

2.1. Quoi qu'il en soit de ses parentés génétiques proches ou lointaines, sur un plan strictement typologique et synchronique, le hourrite est une langue de type agglutinant. De ce point de vue il se distingue nettement des langues indo-européennes, de type flexionnel avec un usage important de la composition, et des langues sémitiques, où la morphologie repose principalement sur la combinaison de squelettes consonantiques fixes et de schèmes vocaliques variables. En hourrite les «mots » sont formés à partir d'une base lexicale, dont le vocalisme est normalement fixe et immuable, et de suffixes, qui viennent se concaténer à la suite les uns des autres. En hourrite affixe veut dire suffixe. Il n'existe pas de préfixe, hormis des prothèses vocaliques pour intégrer phonétiquement des mots étrangers, ni d'infixe, hormis des cas de métathèse. La composition est marginale et réduite à certaines formations, décrites plus loin (\$9.6.). Sur le plan descriptif l'approche du structuralisme américain à base de morphèmes segmentaux convient très bien pour le hourrite. Les «mots » peuvent être tronçonnés en morphèmes, suffixés les uns aux autres dans un ordre qui est prédéterminé à l'avance. Bien qu'il n'ait pas grand chose en commun avec le turc, le hourrite présente un fonctionnement typologique similaire à cette langue, avec cette simplification supplémentaire qu'il n'a quasiment pas d'harmonie

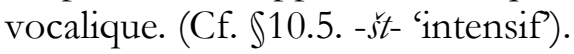

En résumé le hourrite est typologiquement une langue agglutinante suffixante et dépourvue d'harmonie vocalique.

2.2. Les morphèmes du hourrite sont de deux types : des bases lexicales, qui sont normalement le premier élément de la chaine, et ensuite des suffixes, qui viennent s'ajouter aux bases. Décrire le

12 A. FOURNET, «La question des mots et noms mittanni-aryens, légués par les Indo-Iraniens du Mittanni », Res Antiquae 9 (2013), à paraître. 
hourrite revient donc à décrire les différentes classes de bases lexicales, les suffixes, leurs fonctions sémantiques ou syntaxiques, et les concaténations possibles de ces briques élémentaires. Tous les suffixes, ou peu s'en faut, ne peuvent pas apparaitre dans un énoncé ou être utilisés s'ils ne sont pas affixés à une base ${ }^{13}$, et à l'inverse il est rare qu'une base lexicale soit nue. Les bases lexicales et les suffixes sont des virtualités, postulées pour les besoins de la grammaire et de la lexicographie. En pratique on rencontre des «mots", à savoir des concaténations plus ou moins longues de bases et de suffixes. Dans la suite on s'efforcera de distinguer les mots, qui sont des objets susceptibles d'être intégrés en l'état dans une phrase, des bases et des suffixes, qui sont des briques élémentaires inachevées. C'est de cette façon que fonctionne une langue agglutinante, telle que le hourrite. Dans une large mesure, les suffixes ont une fonction ou un sens unique, mais il existe quelques morphèmes tels que $n i$ à valeur multiple (Cf. \$15.5.). Les bases ont normalement un vocalisme stable, mais certains suffixes ont des voyelles variables sans qu'on perçoive quelle différence existerait entre les variantes.

2.3. Les principales classes de bases lexicales sont les noms, les nombres, les pronoms personnels indépendants, les déictiques, les verbes, les adjectifs, les adverbes et les conjonctions. Les principaux types de suffixes sont des formants dérivationnels, des marques de cas, des articles définis ou indéfinis, des pronoms possessifs, des suffixes pronominaux pour la conjugaison verbale, des suffixes pronominaux mobiles, des négations verbales, des temps et modes, des conjonctions enclitiques. Le hourrite n'a pas de genre grammatical. Aucune marque formelle ne permet de prévoir qu'un mot se réfère à un être masculin ou féminin. En général les formes distinguent le singulier du pluriel, mais le procédé pour exprimer le pluriel n'est pas toujours le même. Il n'y a pas de duel, en dehors d'un suffixe verbal particulier, ni de triel. Les bases et les suffixes ont normalement un gabarit phonétique typique qui sera décrit plus loin (\$9.3.).

2.4. Le hourrite est une langue casuelle. La fonction des mots dans la phrase est indiquée par un jeu particulier de suffixes. De ce fait l'ordre des mots est assez libre et ne joue quasiment aucun rôle pour indiquer la fonction syntaxique des mots dans la phrase, contrairement à une langue comme le français ou l'anglais. On peut distinguer deux types de cas : les cas grammaticaux, qui concernent le rôle syntaxique des mots dans la phrase et n'ont pas de contenu sémantique propre, et les cas locaux, qui équivalent aux prépositions du français. Les principaux cas grammaticaux sont l'Absolutif, l'Ergatif, le Génitif et le Datif. Les principaux cas locaux sont le Locatif, l'Allatif, l'Ablatif, le Comitatif et l'Instrumental. Les cas sont similaires dans leur principe à ceux des

13 Dans le vocabulaire bloomfieldien ce sont des "bound forms" : des formes liées, et non des formes libres. 
langues casuelles telles que le latin, le grec, l'allemand ou les langues slaves. En hourrite il n'existe qu'une seule déclinaison valide pour toutes les bases nominales et adjectivales et pour les formes nominales du verbe, à savoir les participes.

2.5. Le hourrite est une langue ergative, et non pas accusative. Cela signifie que le sujet d'un verbe intransitif et l'objet d'un verbe transitif ont la même forme, alors que le sujet d'un verbe transitif reçoit une marque particulière. Dans le premier cas le mot est à l'Absolutif et dans le second cas il est à l'Ergatif. Le système est donc décalé par rapport aux grandes langues de civilisation de l'Europe ou même les langues sémitiques. Ces langues sont de type accusatif : le sujet d'un verbe a toujours la même forme, que le verbe soit transitif ou non, alors que c'est l'objet d'un verbe transitif qui est (éventuellement) différent et reçoit une marque spécifique. Ce décalage explique le recours à une terminologie différente : Absolutif et Ergatif, au lieu de Nominatif et Accusatif. Le hourrite est une langue de type ergatif et cette particularité se retrouve dans le système casuel, la conjugaison, la forme des suffixes de pronoms personnels et les suffixes dérivationnels. En Europe le basque est la seule langue contemporaine ergative. L'ergatif existe également en sumérien, caucasique, etc.

2.6. L'exemple de chaîne suffixale - de mot - qui suit illustre le fonctionnement agglutinant du hourrite et les difficultés récurrentes que le lecteur de cette langue trouve sur son chemin. La tablette (KBo12 44 ii 6) contient une suite de signes cunéiformes dont la translittération standard est <aš-bu-si-ku-un-ni-ni-bi-na-aš$t a>$. Comme son nom l'indique il s'agit d'une tablette (Keilschrift) trouvée à Boğazköy, d'où la référence commençant par KBo. La lecture normalisée de ce mot est ašbušikekunniniwinnašta. Un premier point est que la graphie est défective puisqu'on attendrait $<a \grave{s}-b u-s i s-i k--k u-u n-n i-n i-b i-i n-n a-a \grave{s}-t a>$. Il manque deux signes qui sont soulignés. Le deuxième point est que la syllabe wi est écrite avec le signe $b i$. Il est fréquent que les géminées graphiques soient écrites simples et que les signes pour $w, b$ et $p$ soient presque en variante libre. Il faut prendre l'habitude de ces graphies défectives et variables. Un autre cas d'indétermination concerne les voyelles $i$ et $e$, qui sont sans doute des phonèmes en hourrite mais qui sont en variante graphique permanente l'une de l'autre. A l'intérieur d'un même texte, d'une ligne sur la suivante, le même mot peut être écrit d'une façon ou d'une autre, sans qu'aucune motivation ne soit identifiable. Une autre difficulté est que la segmentation en morphèmes - bases et suffixes - n'a pratiquement aucun rapport avec la segmentation graphique en signes cunéiformes.

2.7. Lire un texte hourrite en cunéiforme suppose donc trois étapes : (1) la translittération brute des signes en alphabet latin, (2) une première concaténation des valeurs syllabiques en mots, (3) une resegmentation des mots en morphèmes qui tienne compte de différents aléas (géminées écrites simples, indistinction des voyelles $i / e$, consonnes $b, p, w$ en variation fréquente). L'exemple de mise en œuvre en fin d'article montre comment s'y prendre. 
2.8. La translittération standard ašhušikkeunniniwinnašta se laisse analyser en morphèmes de la façon suivante :

1. ašb-est une base lexicale de type adjectival : 'haut'. Le mot $a s ̌ b i$ est un adjectif possible et la concaténation peut s'arrêter à ce point.

2. aśbu est un adverbe qui signifie 'vers le haut'. La finale $u$ (\$9.14.) caractérise certains adverbiaux et noms à valeur positionnelle et la concaténation peut s'arrêter à ce point.

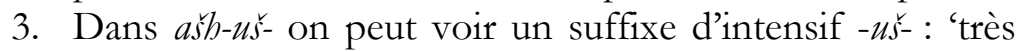
haut'. Le mot ašhuši est un adjectif possible et le mot ašbušu est un adverbe possible.

4. Dans ašbušikk-le suffixe -ikk- nominalise la base suffixée ašhuš- 'très haut' : ašbušikeki est un mot possible 'quelque chose ou quelqu'un qui est très haut' et la concaténation peut s'arrêter à ce point.

5. Dans ašbusikkeunni le suffixe -(u)nni est typique des noms de professions : ašbusikekunni désigne une activité professionnelle en relation avec le 'très haut', et signifie 'prêtre'. La concaténation peut s'arrêter à ce point et fournir une forme de citation qui peut figurer au dictionnaire en tant que nom commun.

6. Dans ašbušikkunnini le suffixe -ni est l'Article Défini au Singulier: ašbusìkkunnini signifie 'le prêtre' au cas Absolutif. La concaténation peut s'arrêter à ce point.

7. Dans ašbusickekunniniwi le suffixe -wi est la marque du cas Génitif : ašbušikekunniniwi signifie 'du prêtre' au cas Absolutif. La concaténation peut s'arrêter à ce point et ce mot peut être Complément du Nom d'un autre mot.

8. Dans ašbušikekunnininna le suffixe -nna est l'Article Défini Pluriel : ašbušikekunnininna signifie 'ceux du prêtre' au cas Absolutif. La concaténation peut s'arrêter à ce point et ce mot peut être intégré dans une phrase en tant que sujet d'un verbe intransitif ou objet d'un verbe transitif.

9. Dans ašhušikekunniniwinnašta le suffixe -šta combine une marque redondante du pluriel $-\xi_{-}^{-}$avec le suffixe du cas Allatif: ašhušikkunnininnašta signifie 'à/vers ceux du prêtre'. La concaténation peut s'arrêter à ce point et ce mot peut être intégré dans une phrase.

Cette chaîne ašbušikekunniniwinnašta attestée dans un texte réel n'est pas au maximum possible car on peut encore lui ajouter des pronoms enclitiques mobiles, par exemple -lla 'P3PL' et des conjonctions enclitiques, par exemple -an 'et'. Par ailleurs une analyse alternative serait d'analyser la base ašbuši- comme étant la graphie défective de ašbušsi 'hauteur', avec un suffixe formant des noms abstraits. Sur le plan sémantique cette analyse alternative ne change pas le sens général, mais en bonne phonétique ašbiššr 'hauteur' est plus correct que *ašbuššri. 


\section{Les principaux documents écrits en langue hourrite}

3.1. Le hourrite est une langue dont l'existence même avait quasiment été perdue. Le premier document qui la fit sortir d'un oubli millénaire est la lettre écrite par le roi du Mittanni, Tušratta, au pharaon égyptien, Amenophis III, connu dans la lettre par son nom royal Neb-Maât-Ra vocalisé $<$ Ni-im-mu-u-ri-i-a $>$, dont une prononciation reconstituée serait donc $*\left[n i m-m u^{?} u-r_{1}^{-P} a\right]$. Cette pièce, trouvée en 1887 et référencée E24, est la seule écrite en hourrite dans tout le corpus des lettres d'El-Amarna. Ecrite en syllabaire cunéiforme, elle pouvait aisément être oralisée, mais il était immédiatement manifeste qu'elle n'était pas écrite dans la langue diplomatique de l'époque, à savoir l'akkadien, hormis l'incipit entièrement rédigé dans cette langue et quelques emprunts. La Lettre du Mittanni resta longtemps le plus long document disponible écrit en langue hourrite. L'étude en est difficile à la fois parce qu'il est monolingue hourrite et parce que les phrases sont souvent longues et leur structure complexe. Les parties les mieux conservées et les mieux comprises de ce document servent de référence linguistique à la présente description. La Lettre comprend 4 tablettes et environ 450 lignes sont conservées. Elle a fait l'objet de différentes études, traduction partielle par Wilhelm ${ }^{14}$ et analyse détaillée par Dietrich \& Mayer ${ }^{15}$. Ce livre récent de Dietrich \& Mayer ambitionnait d'être l'analyse «définitive» de la Lettre du Mittanni.

3.2. Plus récemment, en 1983 puis 1985, lors des campagnes de fouilles estivales, un lot de tablettes bilingues et des fragments a été mis au jour dans des temples à Hattušaš (aujourd'hui Boğazköy/Boğazkale). L'autre langue, écrite dans la colonne de droite, est le hittite. Cette trouvaille a fourni un éclairage nouveau et exceptionnel sur le hourrite, puisque le hittite est mieux compris que ce dernier. La version originale est en hourrite (plutôt archaïsant), sur la colonne de gauche, et la version hittite sur la droite en est une traduction. Il arrive parfois que le texte hittite traduise un commentaire sur le texte hourrite plus que le texte original lui-même, ce qui laisse à penser que des locuteurs hourrites ont pu expliquer à des scribes hittites à la fois les mots du texte et leurs sens. C'est le cas des deux lignes KBo32.14 Vo I 34. Dans l'ensemble le dialecte hourrite attesté dans la Bilingue est archaïque, et en tout cas plus ancien que la traduction hittite, que les hittitologues, tels que Neu, s'accordent à dater vers -1400. La copie autographe de ces textes cunéiformes a été publiée en 1990

14 Cf. W.L. Moran, Les lettres d'El-Amarna. Correspondance diplomatique du pharaon, 1987, Paris, et The Amarna Letters, 1992, London - Baltimore.

${ }^{15}$ M. DIETRICH - W. MAYER, Der hurritische Brief des Dušratta von Mittanni an Amenb otep III. Text-Grammatik - Kopie, 2010, Münster. 
par H. Otten et Chr. Rüster dans le volume 32 des Keilschrifttexte aus Boghåkö̈i (KBo). Une analyse détaillée de ces tablettes a été publiée par E. Neu en 1996 (StBoT35). Ce livre est fondamental pour l'étude du hourrite et sa lecture est vivement recommendable. Ce lot de tablettes correspond à une sorte d'anthologie de la littérature hourrite. D'après la numérotation et les mentions figurant sur les tablettes il est probable que deux œuvres ou deux collections sont mélangées ${ }^{16}$ : d'une part une épopée versifiée en hourrite archaïque : le Chant de la Libération (de la ville d'Ebla), et d'autre part une série d'histoires plus ou moins longues, dont certaines ne sont pas très sages et peuvent être aisément déviées dans une direction obscène. Le hittite traduit la version la plus sage. Neu les a qualifiées de Paraboles mais il n'est pas certain que ce libellé soit le mieux adapté au contenu de certaines d'entre elles.

3.3. Il existe également à Boğazköy un grand nombre de tablettes et de fragments, dont certains traitent de mantique ${ }^{17}$.

3.4. Le texte hourrite le plus ancien, dit Lion d'Urkes, date d'environ -2300. Il s'agit d'une statuette de lion en bronze et d'un galet en calcaire, conservé au Musée du Louvre et ayant servi de " clou » de fondation pour le temple d'Urkeš. Le cunéiforme en est encore presque pictographique. Il contient 25 lignes, gravées sur le galet et moulés sur une tablette incorporée dans le lion en bronze. La langue est en partie similaire par son archaïsme à la Bilingue de Boğąkëy, quoiqu'encore plus ancienne. L'editio princeps a été publiée en 1948 par Parrot \& Nougayrol ${ }^{18}$. Un autre lion est conservé au Metropolitan Museum of Art de New York. Son inscription ne semble pas avoir jamais été publiée.

3.5. Un nombre considérable de mots et noms de personnes hourrites sont attestés dans les textes mésopotamiens et ougaritiques. Une compilation complète de ces éléments reste à faire, même si des compilations partielles existent.

3.6. Les documents hourrites longs sont plutôt rares. Il semble que même dans les régions à majorité hourritophone, la plupart des textes officiels étaient écrits en akkadien, parce que l'usage de l'écrit était d'une certaine façon implicitement lié à la langue akkadienne elle-même, ce qui n'est pas sans rappeler la situation du latin au moyen-âge en Europe ${ }^{19}$. L'akkadien joue le rôle d'hyper-

${ }^{16}$ Cf. E. NEU, Das hurritische Epos der Freilassung (Studien zu den BogazkoyTexten 32), 1996, Wiesbaden, p. 16-20.

${ }^{17}$ Cf. S. DE MARTINO, Die mantischen Texte. Corpus der burritischen Sprachdenkmäler. I. Abteilung, Texte aus Boğazköy, vol. 7, Rome, 1992.

18 «Un document de fondation hurrite», Revue d'Assyriologie 42/1-2 (1948), p. $1-20$

${ }^{19}$ Cf. G. WILHELM, «L'état actuel et les perspectives des études hourrites », dans J.-M. DuRAND (ed.), Amurru 1, Paris, 1996, p. 180. «Ces textes montrent enfin qu'au Mit $[\mathrm{t}] \mathrm{anni}$, également, on se servait comme langue administrative d'un akkadien influencé par le hourrite, comme c'est le cas au pays d'Arrapha. L'espoir de retrouver de nombreux textes en langue hourrite en provenance du 
langue culturelle pour le hourrite et de nombreux mots akkadiens sont utilisés en lieu et place de mots hourrites existants. Par exemple la base an- 'se réjouir', a priori hourrite, a pour synonyme pis- d'origine sémitique. D'autres mots, tels que 'aimer', sont connus sous leur forme akkadienne $t a d-<* d a^{2} d$-, et on ne connait pas l'équivalent hourrite qui devait certainement exister. La capitale du Mittanni ayant été probablement détruite lors de la victoire assyrienne et sa localisation restant inconnue de nous les textes qu'elle renfermait peut-être restent inaccessibles.

\section{Les systèmes d'écriture utilisés}

4.1. Le hourrite est attesté dans plusieurs systèmes d'écriture, dont le principal est le cunéiforme de l'école anatolienne. En raison même de la diversité des systèmes d'écriture utilisés pour le hourrite, cela prit quelque temps au début du $20^{\text {ème }}$ siècle avant que l'on s'aperçût que le hurlili, le mittannien, le subaréen, etc. n'étaient en fait qu'une seule et même langue. En 1932, Friedrich transcrivit la Lettre du Mittanni en la décrivant comme étant un "Subaräische Text", mais dans son travail suivant (publié en 1939), il utilisa le terme désormais usuel "churritisch". Le nom bourrite, sans connotation géographique, s'est imposé pour décrire spécialement la langue en tant que telle, ainsi que nous l'avons noté précédemment. L'ourartéen est écrit en cunéiforme néoassyrien, ce qui explique quelques correspondances phonographémiques inattendues entre lexèmes hourrites et ourartéens.

4.2. La notion d'écriture cunéiforme est en partie trompeuse. Hormis le fait d'utiliser l'argile pour confectionner des tablettes et d'y imprimer des signes, qui est commun à tous les sites et à toutes les écoles, parler «du » cunéiforme au singulier est fictif. En pratique il existe plusieurs écoles scribales qui font des signes un usage différent et il est nécessaire de distinguer ces pratiques, car la manière d'encoder la phonétique du hourrite avec le corpus de signes cunéiformes n'est pas la même, et cela ne concerne pas que la forme des signes. Quatre systèmes d'écriture ont servi à écrire du hourrite : (1) le cunéiforme de l'école anatolienne, et ses diverses variantes, (2) le cunéiforme vieil-assyrien, (3) l'alphabet ougaritique, et enfin (4) les hiéroglyphes louvites (ou anatoliens).

4.3. Aucun des systèmes d'écriture utilisés pour le hourrite ne donne une image véritablement directe et satisfaisante de la langue. Le cunéiforme et le hiéroglyphique louvite sont de type syllabique approximatif. L'alphabet ougaritique d'aspect cunéi-

coeur de l'empire mitannien n'est ainsi plus fondé. [...] Il est donc possible que dans le royaume du Mit[t]anni, sous l'influence de la culture scribale akkadienne dominante, la plupart de la littérature ait été composée en akkadien » [NB : ce point de vue est peut-être trop pessimiste]. 
forme suit une logique précise mais défective où seules les consonnes sont portées à l'écrit. La langue est donc dans une large mesure déduite par recoupements de ces différentes sources. Grâce aux emprunts hourro-ourartéens en arménien on dispose en outre d'une photographie assez claire de la phonologie de l'ourartéen, la langue sœur du hourrite. D'un point de vue méthodologique, une distinction constante doit être faite entre les lectures conventionelles des transcriptions cunéiformes et la réalité phonétique ou phonologique sous-jacente. Et cela vaut pour le hourrite ou toute autre langue du Proche-Orient ancien écrite dans ce système. Nous n'avons pas d'enregistrements directs du hourrite et nous sommes mis dans la situation délicate de reconstruire à partir des données disponibles une image raisonnablement fiable de ce que ces langues ont pu être " pour de vrai ». La même problématique se pose en hittite comme le soulignent Hoffner \& Melchert $^{20}$.

4.4. Le hourrite est principalement attesté en écriture cunéiforme de l'école anatolienne. Les textes en alphabet ougaritique sont rares mais précieux. Ils viennent sans surprise d'Ougarit (Rās Šamra) pour la plupart. Ils apportent un témoignage phonétique d'une grande valeur sur le consonantisme hourrite. Contrairement aux usages akkadiens et hittites, les textes en hourrite mittannien contiennent assez peu de sumérogrammes. Les plus fréquents dans la Lettre du Mittanni sont $\bowtie$ DINGIR 'dieu', $\star$ KUR 'pays', pros MES 'Pluriel' et on peut noter qu'ils ont valeur de déterminatif sémantique et ne sont jamais des segments lexicaux à lire explicitement, contrairement aux usages hittites. Il est également probable pour des raisons phonétiques que

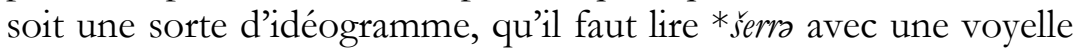
/e/, comme dans šerši 'trône', et non pas /a/ comme en akkadien šarru. La simplicité du cunéiforme hourrite qui recourt surtout à des signes syllabiques élémentaires du type $C \nu$ ou $-\nu C$ est un archaïsme hérité de la première phase d'écriture cunéiforme vieilakkadienne. Cette simplicité se retrouve dans les colonnes de la Bilingue de Boğąkëy écrites en hourrite mais pas dans celles écrites en hittite, qui utilisent plus abondamment les idéogrammes sumériens. Ce fait en soi indique que la version hourrite est plus ancienne que la traduction hittite.

4.5. En toute rigueur, comme il existe plusieurs écoles cunéiformes, il faudrait préciser dans la translittération à laquelle elle se réfère. Dans le domaine hourro-ourartéen, il en existe quatre: l'école vieil-assyrienne, qui distingue des signes avec sourdes et sonores, l'école anatolienne, qui distingue des simples et des géminées graphiques, avec deux variantes hittite et mittannienne qui diffèrent par quelques détails intéressants, et l'école néoassyrienne, qui s'applique à l'ourartéen. Dans l'ensemble ces

${ }^{20}$ H.A. HofFner JR. - H.C. MELCHERT, A Grammar of the Hittite Language, Part I: Reference Grammar, Winona Lake (Indiana), 2008, p. 10. 
écoles divergent surtout sur la valeur des signes relatifs aux fricatives et affriquées coronales, à savoir les phonèmes pour lesquels les langues sémitiques divergent le plus entre elles. Les autres signes sont normalement identiques. En l'absence de toute indication il est possible de s'appuyer sur certaines particularités du cunéiforme lui-même: un texte qui pratique la gémination graphique est anatolien, une abondance de sumérogrammes indique

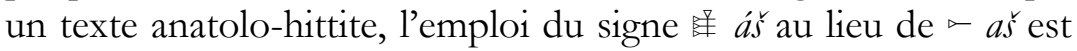
vieil-assyrien et se rencontre par exemple à Urkeš et Qaṭna. Il ne semble pas exister d'étude paléographique sur ces questions.

4.6. L'école anatolienne, qui recourt à la gémination graphique pour noter l'opposition sourde $\sim$ sonore, est représentée dans la Lettre du Mittanni, la plupart des villes de Syrie, les textes tardifs d'Arraphi et Nuzi, et à Hattušaš. L'autre école, utilisée par les Assyriens entre -2300 et -1700 , apparait à Mari, en vieux babylonien (avant -1700), à Arraphi et Nuzi (avant -1400), et aussi à Kanesh avant $-1700^{21}$. Les consonnes finales et initiales ne sont jamais géminées et ne montrent donc aucune distinction explicite.

On sait par le biais de l'alphabet ougaritique que le hourrite n'admet que des sourdes à l'initiale mais que la distinction sourde sonore est maintenue en finale de mot. La nature des traits phonologiques que le système graphique distingue par la gémina-tion reste controversée et discutée. Concernant le hittite HoffnerMelchert ${ }^{22}$ font observer que «there is no consensus as to whether the phonetic contrast in attested Hittite is one of voicing or of some other feature such as "fortis" vs. "lenis" or "aspirated" vs. "unaspirated" ». Leur point de vue est néanmoins que "for the sake of simplicity we here describe the contrast in stops as one of voicing, but we do not mean thereby to take a definitive stance on this issue $»^{23}$. C'est le point de vue qui est retenu ici.

4.7. Laroche ${ }^{24}$ considérait que la gémination graphique correspond à une opposition phonétique de voisement: "L'écriture alphabétique de Rās Šamra apporte un précieux concours; elle seule, en effet, permet de discriminer sans hésitation les sourdes et les sonores. Elle a confirmé l'opposition pertinente de $p$ à $b$, de $t$ à $d$, etc., que les syllabaires occidentaux représentent par l'artifice de la gémination: $-b b$ - ou $-p p-=p$, en face de $-b$ - ou $-p-=b$, $-t t-=t$, etc. ». Un exemple est le théonyme Teřšsub aux cas Absolutif et

${ }^{21}$ Cf. E.A. SPEISER, Introduction to Hurrian (Annual of the American Schools of Oriental Research 20.), New Haven (CT), 1941, p. 40-41, et I.M. DiAKONOV, Jazykei drevnej perednej Aziji, Moscou, 1967, p. 120.

22 H.A. HoffNer Jr. - H.C. MelCherT, op. cit. (n. 20), p. 35 : « Il n'y a pas consensus sur la question de savoir si l'opposition phonétique en hittite repose sur le voisement ou sur quelque autre trait tel que "fortis" vs. "lenis" ou "aspiré" vs. "non aspiré" ».

${ }^{23}$ IBID., p. 35 : «Par simplicité nous décrivons ici l'opposition des occlusives comme étant le voisement, mais cela n'entraine pas que nous prenons une position définitive sur cette question ».

${ }^{24}$ LAROCHE, op. cit. (n. 1), p. 22. 
Datif : Teršsub $\sim$ Te šš sppe, respectivement écrits : $[\mathrm{t} \underline{\mathrm{t}} \mathrm{b}] \sim[\mathrm{t} \underline{\mathrm{t}} \mathrm{p}]$ en ougaritique. On peut noter que le hittite écrit en ougaritique est cohérent avec le hourrite : <Šu-up-pí-lu-li-u-ma> s'écrit [t p 11 $\mathrm{m}]$. Laroche attribue le mérite de cette théorie à Speiser: «les nouveaux textes confirment largement la doctrine de Speiser, selon laquelle les sonores et les sourdes intervocaliques de l'alphabet répondent à des graphies simples et géminées dans les syllabaires de Boğ., RS et Mit. [Boğazköy, Rās Šamra et Mittanni] $\gg^{25}$ Ce principe est en effet décrit par Speiser ${ }^{26}$. En hittite il fut découvert par Sturtevant, d'après une suggestion originellement formulée par Mudge ${ }^{27}$.

4.8. Le hourrite écrit en alphabet ougaritique n'utilise que les lettres pour les sourdes et les sonores, à l'exclusion de toutes les emphatiques $: h, h, t, z, s, s, q$ ne sont pas utilisées. Cette situation est cohérente avec l'adaptation des emphatiques de l'akkadien sous forme de sonores en hourrite, notées simples en cunéiforme de l'école anatolienne. Cela suggère que l'akkadien et les dialectes sémitiques mésopotamiens avaient des emphatiques sonores.

\section{Approche de la phonologie hourrite}

5.1. Il est possible de reconstituer une image sans doute fiable de la phonétique réelle de la langue hourrite par recoupement entre les différentes sources. Certaines d'entre elles comme l'arménien ou l'indo-iranien sont très fiables. D'autres comme l'indo-européen ou le protosémitique sont plus discutables, car elles intègrent elles-mêmes une part d'hypothèses. Quoi qu'il en soit, dans une optique principalement philologique, la phonétique reconstruite n'a pas réellement d'importance. La question est plus de définir une oralisation conventionnelle des translittérations. La phonétique reconstruite intéresse plutôt les comparatistes.

5.2. La prosodie du hourrite telle qu'elle se manifeste dans l'écriture plene des voyelles n'a été que très peu étudiée. Il semble exister plusieurs types d'accentuation et l'accent semble pertinent et mobile ${ }^{28}$. Les idées développées par Wilhelm sur cette question sont très insuffisantes ${ }^{29}$.

25 E. LAROCHE, «Documents en langue hourrite provenant de Ras Shamra » in C. SCHAEFFER (ed.), Ugaritica 5: nowveaux textes accadiens, hourrites, et ugaritiques des archives et bibliothèques privées d'Ugarit, commentaires des textes historiques (Mission de Ras Shamra 16 = Institut français de Beyrouth, Bibliothèque archéologique et historique 80), Paris, 1968, p. 528.

${ }^{26}$ SPEISER, op. cit. (n. 21), p. 35-36.

${ }^{27}$ Cf. HOFFNER - MELCHERT, op. cit. (n. 20), p. 35.

${ }^{28}$ Cf. FOURNET - BOMHARD, op. cit. (n. 10), p. 15-17.

${ }^{29}$ G. WILHELM, « Hurrian », in R.D. WOODARD (ed.), The Ancient Languages of Asia Minor, Cambridge, 2008, p. 86, \$3.4. 
5.3. Sur le plan graphémique le corpus de signes cunéiformes comprend les types suivants :

- des voyelles : a, i, e, u, ú.

- des signes Cv, vC avec des labiales : p, b, m, w.

- des signes $\mathrm{Cv}, \mathrm{vC}$ avec des dentales : $\mathrm{t}, \mathrm{d}, \mathrm{n}$.

- des signes $\mathrm{Cv}, \mathrm{vC}$ avec des vélaires : $\mathrm{k}, \mathrm{g}$, h. Le graphème $<\mathrm{h}>$ est écrit $<\mathrm{h}>$ (h lunulé) par les sémitologues mais cette précision est inutile en cunéiforme hourrite, car il n'existe pas d'autre fricative gutturale de ce type dans cette langue.

- des signes $\mathrm{Cv}, \mathrm{vC}$ avec des liquides $\mathrm{r}$, $\mathrm{l}$. Il peut être utile de rouler le $[\mathrm{r}]$ pour le distinguer de la vélaire $h$.

- un signe pour $y a$, qui est une ligature des signes $<\mathrm{i}-\mathrm{a}>$, mais pas d'autres signes $\mathrm{Cv}, \mathrm{vC}$ impliquant $/ \mathrm{j} /$ 'yod'. Les signes $i$ et $e$ notent sans doute à la fois $/ \mathrm{i} /$ et $/ \mathrm{ji} /$, / e/ et $/ \mathrm{je} /$.

Outre le fait que le hourrite semble présenter une variabilité interne élevée pour les voyelles $i$ et $e$, le cunéiforme lui-même est ambigu et les signes $r$, $b$, nə ne distinguent pas entre $i$ et $e$. Les signes ab et wa valent pour les quatre voyelles $a, e, i, u$. L'école anatolo-hittite a créé différentes ligatures de wa, qui suppriment l'ambiguïté. Le signe ah est ambigu, n'a jamais été désambiguïsé, et en général on lui assigne la même voyelle que le signe qui précède, ce qui paraît raisonnable.

5.4. Le système ne permet pas de noter l'arrêt glottal explicitement. L'existence de séquences ${ }^{2} v$ et $v^{\text {? }}$ en hourrite est probable en raison de nombreux hiatus. Il est possible que la différence entre $u$ et $\dot{u}$ soit que $\left\langle u\right.$ note $\left[{ }^{3} \mathrm{u}\right]$ alors que $u$ note $[\mathrm{u}]$ et $[\mathrm{w}]$. Le signe $u$ est utilisé en akkadien pour allonger la voyelle $u$ et ne comporte pas d'arrêt glottal implicite.

5.5. Le problème de fond concerne les trois séries de signes cunéiformes translittérés avec $z, s$ et $r$. Implicitement ces graphies suggèrent une prononciation respectivement sifflante: $[\mathrm{z}]$, [s], et chuintante: $[\mathrm{J}]$. Il est certain que ces prononciations implicites sont fausses. A haute époque, lorsque l'akkadien a été écrit pour la première fois, la série-z était affriquée $[\mathrm{dz}]$, la série-s était utilisée pour les fricatives latérales *ś héritées du protosémitique, et la série- $s$ notait des sifflantes simples [s]. C'est ce que montrent toutes les langues non-sémitiques, comme le hittite ou les mots de substrat hourro-ourartéens en arménien. Les graphies $\approx s$ et $\breve{s}$ propagent de façon abusive les reflets néo-hébreux des phonèmes protosémitiques *dz, *ś et *s dans tout le cunéiforme, et par conséquent dans toutes les langues écrites de cette façon. Ce problème n'est pas (trop ?) gênant quand on reste à l'intérieur du domaine sémitique mais il met la phonétique des coronales de travers pour les autres langues : hourrite, hittite, etc. ${ }^{30}$

30 Cf. L. Kogan, "Proto-Semitic Phonetics and Phonology», in S. WenINGER, The Semitic Languages: An International Handbook, Berlin, 2011, p. 54150, en particulier $\$ 1.3 .2$. 
5.6. Il faut donc gérer une situation où la valeur implicite de trois séries de signes cunéiformes, à savoir les séries $\%, s$ et $r$, sont assurément fausses. Certains hittitologues ont opté pour une suppression du haček sur la série- $s$, qu'ils translittèrent avec un simple $s$, étant donné que le $s$ hittite est le continuateur du $s$ indoeuropéen. En hittite on ne rencontre normalement que les deux séries z et $\check{s}$, et jamais la série $s$. La suppression du haček ne pose pas de problème si l'on ne fait que du hittite. Mais elle crée un problème pour le hourrite mittannien qui utilise les trois séries. En particulier le hourrite mittannien utilise de façon pertinente la série- $s$ relative aux fricatives latérales dans des emprunts qui en ont effectivement d'après le proto-sémitique. Il semble difficile de réformer les translittérations traditionnelles sans créer en ricochet d'autres problèmes et d'autres confusions. D'un point de vue pratique le plus simple est d'oraliser la série-z avec des affriquées $[\mathrm{dz}]\langle\mathrm{z}\rangle$, [ts] $\langle\mathrm{zz}\rangle$, la série- $s$ et la série- ${ }^{s}$ avec [s] et [S] tout en gardant à l'esprit que cette oralisation est fausse du point de vue de la phonétique reconstruite.

5.8. Il est possible que la série- $\breve{s}$ serve en hourrite à noter deux séries de phonèmes différents car le morphème $s$ est écrit avec la lettre ougaritique $\underline{t}$ pour l'Ergatif mais $s$ pour le Pluriel. Le Pluriel est une sonore $-\nu \Sigma^{2} \nu$-, alors que l'Ergatif est une sourde $-\nu s^{2} \nu^{2} \nu$. Pour l'étude des textes en cunéiforme mittannien et anatolo-hittite ces questions phonographémiques n'ont pas d'importance.

5.9. Le système de phonèmes le plus simple qu'on puisse attribuer au hourrite est le suivant:

\begin{tabular}{|l|c|c|c|c|c|c|}
\hline & Labiale & Dentale & Affriquée & Sifflante & Vélaire & Vélaire \\
\hline Sonore & $\mathrm{b}$ & $\mathrm{d}$ & {$[\mathrm{dz}]$} & {$[\mathrm{z}]$} & $\mathrm{g}$ & {$[\gamma]$} \\
\hline Sourde & $\mathrm{p}$ & $\mathrm{t}$ & {$[\mathrm{ts}]$} & {$[\mathrm{s}]$} & $\mathrm{k}$ & {$[\mathrm{x}]$} \\
\hline Nasale & $\mathrm{m}, \mathrm{mm}$ & $\mathrm{n}, \mathrm{nn}$ & & & & \\
\hline Liquide & & $1, \mathrm{ll}, \mathrm{r}, \mathrm{rr}$ & & & & \\
\hline Autre & $\mathrm{w}$ & & & $\mathrm{y}$ & & \\
\hline
\end{tabular}

Inventaire minimal d'après Laroche (1980: 23-24)

Ces phonèmes sont écrits de la façon suivante dans l'école anatolienne du cunéiforme en usage au Mittanni et chez les Hittites :

\begin{tabular}{|l|c|c|c|c|c|c|}
\hline & Labiale & Dentale & Affriquée & Sifflante & Vélaire & Vélaire \\
\hline Sonore & $\mathrm{b}$ & $\mathrm{d}$ & $\mathrm{z}$ & $\check{\mathrm{s}}$ & $\mathrm{g}$ & $\mathrm{h}$ \\
\hline Sourde & $\mathrm{p}(\mathrm{p})$ & $\mathrm{t}(\mathrm{t})$ & $\mathrm{z}(\mathrm{z})$ & $\mathrm{s}(\check{\mathrm{s}})$ & $\mathrm{k}(\mathrm{k})$ & $\mathrm{h}(\mathrm{h})$ \\
\hline Nasale & $\mathrm{m}, \mathrm{mm}$ & $\mathrm{n}, \mathrm{nn}$ & & & & \\
\hline Liquide & & $\mathrm{l}, \mathrm{ll}, \mathrm{r}, \mathrm{rr}$ & & & & \\
\hline Autre & $\mathrm{w}$ & & & $\mathrm{y}$ & & \\
\hline
\end{tabular}

Système graphique du cunéiforme anatolien 
5.10. La gémination graphique qui note les sourdes ne peut être mise en œuvre qu'à l'intervocalique. A l'initiale le hourrite semble avoir perdu le contraste entre les sourdes et les sonores que l'ourartéen a conservé. Les seuls mots qui ont des sonores à l'initiale en hourrite ougaritique sont des mots sémitiques. Les scribes ont rétabli la sonore dans les mots comme Daqita qu'ils ont perçus comme sémitiques. En finale la marque contrastive de voisement existe d'après les données ougaritiques mais elle ne peut pas être notée explicitement en cunéiforme.

Les phonèmes $r, l, n$ et $m$ peuvent être écrits géminés $r r, l l, n n$ et $m n$, ce qui correspond à une réalité phonologique. Il existe au moins un exemple en alphabet ougaritique de $<$ nn $>$ géminé. La gémination pour les autres phonèmes est un artifice graphique.

5.11. Après $n$ et $l$, il arrive que $\zeta$ soit écrit z, c'est-à-dire que la sifflante simple $\check{s}[\mathrm{~s}]$ est affriquée [dz]. Par exemple itkalzi est soit le dérivé abstrait *itkal[išsi ou l'intensif $*$ itkal $\left[u s^{\prime}\right]$ si avec chûte d'une syllabe. Le déictique manš- est aussi attesté man₹--

5.12. Le cas le plus problématique est le phonème $* b$ à l'initiale des mots, qui est reclassé tantôt en $p$ tantôt en $w$ : ourartéen baban 'montagne' mais hourrite pabani ou wabani. Ce problème concerne tous les mots qui avaient une labiale sonore $* b$ à l'initiale en proto-hourro-ourartéen. Pour les mots avec alternance graphique initiale $\mathrm{p} / \mathrm{w}$ on peut adopter comme translittération conventionnelle la lettre $\beta$. Les autres sonores initiales se sont simplement assourdies et sont écrites avec la sourde de même articulation. Elles ne montrent pas d'alternance graphique. L'articulation de $b$ est très spirante et peut même aller jusqu'à $y$ : abi, awi, ayi 'visage'. De même sizwe, sizye 'eau'. J'ai proposé que le mot arménien ait 'joue' dérive de l'hourro-ourartéen abi 'visage'.

5.13. L'existence d'un arrêt glottal / / en hourrite est probable, mais reste difficile à établir de façon définitive. On peut ne pas tenir compte de cette question pour la philologie.

Le cas de la géminée graphique wn est une question ouverte: s'agit-il de $[\mathrm{ww}]$ ou de $\left[{ }^{\mathrm{w} w}\right]$ ?

5.14. En terme de voyelles le hourrite possède de façon certaine une opposition entre $/ \mathrm{a} / \mathrm{h} / \mathrm{u} /$ et la paire $\langle\mathrm{i}\rangle,\langle\mathrm{e}\rangle$. Les voyelles $i$ et $e$ alternent fréquemment et la distinction est faible. Certains $a$ suivis des consonnes $r, l, n$ et $h$, passent à $e$ : talmi $=$ telami 'grand', AN > (emprunt) eni 'dieu', akkadien šarru $>$ * särrišse $>$ serrišse, šerše 'royauté, trône', akkadien maharu > meh- 'se présenter devant (un dieu)'. Une explication possible est que la voyelle $a$ accentuée passe à $e$ quand elle est suivie des consonnes $r, l, n$ et $h$. Ce phénomène peut être indiqué avec $<\ddot{a}>$ : šärri. Ce changement suggère que le hourrite avait un accent de hauteur (pitch). A noter aussi le cas du mot hourrite eari <e-a-ar-ra> 'lion', qui se laisse rapprocher de *'ari 'lion', avec une sorte de fracture de la voyelle [ea]. 
5.15. Il est possible que le hourrite ait eu un phone [ə] dans certaines syllabes inaccentuées. Certains mots présentent des alternances graphiques telles que Kumarbi $=$ Kumurbi, Kušub $=$ Kušah, $e b-r i=e$-pir $=e$-bar-, etc., ce qui laisse à penser que le timbre de la voyelle est assez neutre. Cette suggestion remonte (au moins) à Diakonov et, encore plus tôt à Speiser, qui avait évoqué la possibilité de « voyelle inorganique ».

\section{Le févo-hourrite}

6.1. Les études hourritologiques peuvent être réparties en deux tendances: une tendance hittitologique et une autre à laquelle je donne le nom de févo-hourrite. La tendance hittitologique est celle qu'avaient adoptée Thureau-Dangin, Speiser, Laroche, Neu, etc., et qui est prise comme référence ici. D'autres chercheurs, Bush, Campbell, Wilhelm, Wegner, Giorgeri, Girbal, etc., ont adopté une autre approche de la phonétique reconstruite du hourrite et ont considéré comme valide l'idée que le hourrite aurait des phonèmes $f, v$ et aussi $o$.

6.2. L'approche févo-hourrite postule l'existence de phonèmes $f, v$ et $o$ en hourrite et considère que la gémination graphique correspond à une gémination réelle. Cette approche est, à mon humble avis, erronée sur chacun de ces points.

6.3. La postulation de phonèmes $f$ et $v$ en hourrite remonte à Bork, un orientaliste très "créatif", au mauvais sens du terme. Cette invention surinterprète les graphies de la façon suivante: par exemple le mot 'montagne' est attesté <pa-pa-ni>, <wa-wa$\mathrm{ni}>,<\mathrm{pa}-\mathrm{ab}-\mathrm{ni}>$. L'alternance $\mathrm{p} / \mathrm{w}$ est prise pour l'indication que la phonétique sous-jacente serait *faban avec $f$ initial, un phonème que le cunéiforme ne peut pas noter directement. L'ourartéen baban, et le grec Bouvos, montre que cette hypothèse ne tient pas. Cette alternance est due au reclassement sans doute variable suivant les locuteurs de l'ancienne initiale sonore $* b$ devenue impossible en hourrite, attesté à date historique. De même le suffixe possessif 'P1SG', écrit <ib-wə>, est censé être *-iff- dans cette approche févo-hourrite. Le mot akkadien pedū 'clément', d'une racine sémitique $p d w$ ( $p d h$ en hébreu avec mater lectionis), est emprunté en hourrite et écrit <pe-du-ub-wa> pedunwa dans la Bilingue de Boğąköy. La phonétique *peduffu est inadéquate. Il y a aucune raison d'interpréter la graphie $\langle\mathrm{vb}-\mathrm{wv}\rangle$ comme étant l'indication de -ff-. Il n'y a aucune raison de postuler que les suffixes $w i$ et $w a$ seraient $* v i$ et $* v a$. Les données comparatives ourartéennes et akkadiennes montrent que ces phonèmes $f$ et $v$ sont des hypothèses, dépourvues de fondement réel.

6.4. Le févo-hourrite postule également que le hourrite aurait un phonème ${ }^{*} o$. Cette invention repose sur un seul texte : la Lettre du Mittanni. Dans sa thèse de 1964 Bush a émis l'idée que les deux signes cunéiformes gu et ku auraient été spécialisés par les scribes 
du Mittanni pour écrire les syllabes $\mathrm{ku} / \mathrm{gu}$ et ${ }^{*} \mathrm{ko} /{ }^{*}$ go respectivement $^{31}$. Cette théorie postule que les signes $u$ et $\dot{u}$ seraient utilisés pour distinguer $*_{O}$ et $u$ respectivement par les scribes du Mittanni. Il convient de souligner que cette théorie n'est valide que pour ce texte, et lui seul, et que ce « système » graphique n'est appliqué nulle part ailleurs. L'analyse distributionnelle des signes à l'intérieur même de la Lettre montre que cette hypothèse ne tient pas. Par exemple on ne rencontre jamais ku à l'initiale des mots, mais seulement $g u$. Le mot $u-u-m i-i-n i$ 'pays' est un des rares mots à deux voyelles plene en hourrite. Cette théorie le reconstruit *ōmini. Ce mot est certainement un emprunt indo-iranien: vieil indien bhümi 'terre, monde, sol' < *bbuHmiH 'ce qui est', d'après la racine indo-européenne $* b b u H$ - 'être, croître'. Le vocalisme du vieil indien $[\bar{u}]$ montre que la phonétique *ōmini n'a aucune validité. Ce mot a deux voyelles plene parce qu'il est emprunté et il repose sur * [ümīni]. Les mots de substrat en arménien indiquent que le phonème $/ \mathrm{u} /$ était $[\mathrm{u}]$ lorsque bref mais [ō] lorsque long. Cette répartition vaut pour l'ourartéen mais elle s'applique - peut-être aussi, voire certainement - au hourrite. Aucune base comparative ne permet de confirmer l'existence de deux phonèmes /u/ et $*_{\mathrm{O}}$, qui pourraient en outre être long et bref. L'hypothèse que la Lettre du Mittanni serait le seul document où existerait une distinction entre $/ \mathrm{u} /$ et un hypothétique $5^{\text {ème }}$ phonème ${ }^{*}$ o ne tient pas. La distinction supposée exister entre ki et gi dans la Lettre est aussi infondée que celle entre ku et $g u$. Le verbe <gi-pa-> (Mitt. II 16, 20, 22, 54, 63) est aussi écrit <ki-i-pa-> (Mitt. II 103, 104). Il signifie 'déposer' et se réfère aux cadeaux envoyés par Tušratta. Il n'y a qu'un seul verbe kib-, écrit à la fois <gi-pa- $>$ et $<$ ki-i-pa- $>$. Cette théorie est de fait réfutée par le texte même où elle prétend puiser sa source.

6.5. L'approche févo-hourrite prétend aussi que la gémination graphique serait une gémination réelle. Il apparaît que le hourrite n'admet pas d'autre géminée réelle que $n n, l l, r r$ et $m m$. Ce fait est établi grâce aux emprunts d'origine suméro-akkadienne dans lesquels des géminées réelles sont adaptées en hourrite sous forme de prénasalisées :

- akkadien Araššbu > hourrite Aranzah 'le Tigre',

- sumérien kukkal > hourrite kungalli 'une sorte de mouton',

- akkadien massäru 'protection' > hourrite manzadubli 'policier',

- akkadien suluppu > hourrite zilumba 'datte (fruit)',

- akkadien tabbihu > hourrite zambahu-nni 'boucher',

- akkadien Purattu $>$ hourrite Puranti 'l'Euphrate',

- sumérien Piriggal $>$ hourrite Piringir 'une déesse, lit. la grande lionne'. Ce mot présente en outre un rhotacisme du $l$ qui n'est pas d'origine hourrite (peut-être hattie ?).

${ }^{31} \mathrm{~F}$. BusH, A Grammar of the Hurrian Language, Ph.D. dissertation, Brandeis University, Department of Mediterranean Studies, 1964, p. 22. 
Il n'existe pas d'exemple de géminée akkadienne qui soit rendue par une géminée graphique en hourrite. Qu'elles soient labiales, dentales, vélaires, occlusives ou sifflantes, les géminées étrangères -CC-sont dissimilées sous forme de prénasalisées -nC-, -mC-, lorsqu'elles empruntées en hourrite.

6.6. En résumé, les différentes hypothèses sur lesquelles repose le févo-hourrite : phonèmes $*_{\mathrm{f}},{ }_{\mathrm{v}}$, ${ }^{*} \mathrm{O}$, géminées réelles et non pas artifices graphiques, peuvent être démontrées fausses. Bien qu'elle soit utilisée par une majorité de hourritologues, et qu'elle semble même être de plus en plus employée depuis une décennie ou une quinzaine d'années, elle doit être tenue pour invalide. Il est en outre scandaleux et anti-scientifique d'utiliser cette phonétique févo-hourrite sans préciser au moyen d'un astérisque qu'elle est hypothétique, et de l'utiliser en lieu et place de la translittération conventionnelle du cunéiforme. On rencontre même des auteurs qui translittèrent de façon encore plus abusive le mot 'montagne' $<$ fa[sic]-ba-ni> au lieu de <wa-ba-ni $>$ en propageant à rebours dans le cunéiforme cet ensemble de postulations douteuses, ce qui achève de créer la plus grande confusion sur la phonétique et la phonologie du hourrite, et celles des signes cunéiformes.

6.7. Le plus simple pour reformater du févo-hourrite est de remplacer $*^{*}$ par $u, *^{*} / *^{*} v$ par $w, *_{f f}$ par $w w$ et à l'initiale $* f$ par $\beta$. Il est également fréquent que $\check{s}$ intervocalique soit écrit $* \tilde{z}$, par les auteurs à tendance févo-hourrite. En règle générale il n'est plus possible de récupérer les données cunéiformes authentiques à partir du formatage févo-hourrite, les auteurs pratiquant le févohourrite ne fournissant pas de translittération fidèle la plupart du temps, bien que les moyens modernes de traitement de texte par ordinateur permettent de citer les sources directement en cunéiforme de plus en plus facilement. Voir l'exemple en fin d'article.

\section{Bibliographie conseillée}

La bibliographie conseillée dépend naturellement des objectifs recherchés. Dans le domaine du comparatisme hourro-ourartéen, on peut recommander :

I.M. Diakonov, Jazyki drevnej perednej Aziji, Moskva, 1967.

I.M. DiakONOV, Hurritisch und Urartäisch, München, 1971.

Les noms de M. SALVINI et M.L. HAČIKYAN semblent les auteurs actuels les plus pertinents sur l'ourartéen. Sur le kassite, on peut lire :

T. SCHNEIDER, «Kassitisch und Hurro-Urartäisch, Ein Diskussionsbeitrag zu möglichen lexikalischen Isoglossen », Altorientalische Forscbungen 30 (2003), p. 372-381. 
A. FOURNET, "The Kassite Language in a Comparative Perspective with Hurrian and Urartean », The Macro-Comparative Journal 2/1 (2011), p. 1-19.

Sur la question du superstrat indo-iranien au proche-orient :

M. Mayrhofer, Die Indo-Arier im alten Vorderasien, Baden-Baden, 1966,

M. Mayrhofer, Die Arier im vorderen Orient, ein Mythos?, Wien, 1974.

M. Mayrhofer, «Welches Material aus dem Indo-arischen von Mitanni verbleibt für eine selektive Darstellung? ", in E. NEU (ed.), Investigationes philologicae et comparativae: Gedenkschrift für Heinz Kronasser, Wiesbaden, 1982, p. 72-90.

A. Fournet, «La question des mots et noms mittanni-aryens, légués par les Indo-Iraniens du Mittanni », Res Antiquae, 2013, (à paraître).

Sur les mots de substrats en arménien :

J.A.C. GreppIN : Nombreux articles dans Aramazd.

Les mots de substrat hourro-ourartéens en kurde semblent une terra incognita. Mis à part quelques approches faites par des « amateurs » sur internet, il ne semble pas exister de relevé des mots kurdes pour lesquels une origine hourro-ourartéenne soit recevable. Sur les questions relatives à la problématique dite euphratique, on peut consulter les articles dans le JIES.

Sur le hourrite proprement dit, il faut lire les volumes des Studies on the Civilization and Culture of Nuri and the Hurrians (SCCNH), en particulier le volume 10, un des plus linguistiques de la série. Les ouvrages ou articles les plus intéressants par ordre de parution :

E. SPEISER, Introduction to Hurrian (Annual of the American Schools of Oriental Research 20), New Haven (CT), 1941. Ce livre est plus ou moins dépassé mais il mérite toujours d'être lu pour le questionnement, plus que pour les réponses.

I.J. GELB, Hurrians and Subarians, Chicago (IL), 1944.

F. Bush, A Grammar of the Hurrian Language, Ph.D. dissertation, Brandeis University, Department of Mediterranean Studies, 1964. La thèse se présente comme une continuation de Speiser (1941) mais elle s'en écarte (à mon humble avis pour le pire). Initiateur du févo-hourrite.

J. FrIEDRICH, «Churritisch », in B. SPULER (ed.), Altklein-asiatische, Leiden, 1969, p. 1-30. Sérieux mais plus ou moins dépassé.

E. LAROCHE, Glossaire de la langue hourrite (= Revue Hittite et Asianique 34/35), Paris, 1981. Toujours utile même si une mise à jour serait éminemment opportune. 
E. Neu, Das hurritische Epos der Freilassung (Studien zu den Bogazkoy-Texten 32), Wiesbaden, 1996. A lire en premier. Peut servir de manuel d'apprentissage du hourrite, malgré quelques lacunes dans l'analyse et des erreurs de translittération du cunéiforme, ainsi qu'il apparaittra en fin d'article.

M. Giorgieri, "Schizzo grammaticale della Lingua Hurrica», dans La civiltà dei Hurriti, La Parola del Passato, Vol. 55. Napoli, 2000, p. 171-277. L'ouvrage est une sorte de point général, à tendance encyclopédique, sur la question hourrite en langue italienne. Applique le févo-hourrite.

I. WeGNER, Hurritisch: eine Einfübrung, (2 ${ }^{\text {ème }}$ éd.), Wiesbaden, 2007. Le livre est surtout intéressant pour sa deuxième partie consacrée à l'analyse de textes en hourrite. Applique le févo-hourrite.

G. WILHELM, «Hurrian», in R.D. WoOdARD (ed.), The Ancient Languages of Asia Minor, Cambridge, 2008, p. 81-104. Applique le févo-hourrite.

M. Dietrich - W. MAYER, Der hurritische Brief des Dušratta von Mittanni an Amenbotep III. Text - Grammatik - Kopie, Münster, 2010. Translittération incohérente, mais néanmoins utile pour les données. Ne peut pas servir de manuel d'apprentissage.

\section{Abréviations}

$\begin{array}{ll}\text { ABL. } & \text { 'cas Ablatif } \\ \text { ABS. } & \text { 'cas Absolutif } \\ \text { ALL. } & \text { 'cas Allatif } \\ \text { COM. } & \text { 'cas Comitatif } \\ \text { DAT. } & \text { 'cas Datif } \\ \text { DEF. } & \text { 'Article défini' } \\ \text { DESI } & \text { 'forme Désidérative' } \\ \text { ENC } & \text { 'Conjonction enclitique' } \\ \text { ERG. } & \text { 'cas Ergatif } \\ \text { FUT } & \text { 'temps verbal Futur' } \\ \text { GEN. } & \text { 'cas Génitif } \\ \text { GER } & \text { 'gérondif } \\ \text { HCN } & \text { 'hourrite classique normalisé' } \\ \text { INDI } & \text { 'mode Indicatif } \\ \text { INST. } & \text { 'cas Instrumental' } \\ \text { INT } & \text { 'Intransitif } \\ \text { LOC. } & \text { 'cas Locatif } \\ \text { NEG } & \text { 'Négation verbale' } \\ \text { OPTA } & \text { 'mode Optatif } \\ \text { PART } & \text { 'Participe' } \\ \text { PEM } & \text { 'pronom enclitique mobile' } \\ \text { PL } & \text { 'Pluriel' } \\ \text { PLI } & \text { 'pronom libre indépendant' } \\ \text { POSS } & \text { 'suffixe pronominal possessif } \\ & \end{array}$




$\begin{array}{ll}\text { PRES } & \text { 'temps verbal Présent' } \\ \text { PRET } & \text { 'temps verbal Prétérit' } \\ \text { P1SG, P1PL } & \text { '1 ière personne du singulier, du pluriel' } \\ \text { P2SG, P2PL } & \text { '2 }{ }^{\text {ème }} \text { personne du singulier, du pluriel' } \\ \text { P3SG, P3PL } & \text { '3 }{ }^{\text {eme }} \text { personne du singulier, du pluriel' } \\ \text { SG } & \text { 'Singulier' } \\ \text { SPAI } & \text { 'suffixe pronominal actif de l'Indicatif } \\ \text { SPOD } & \text { 'suffixe pronominal de l'Optatif et du Désidératif } \\ \text { TC } & \text { 'translittération des signes cunéiformes' } \\ \text { TRA } & \text { 'Transitif' }\end{array}$

\section{La morphologie nominale}

9.1. Les bases et lexèmes de type nominal peuvent être classés en cinq grandes catégories :

- des noms communs. Ils se terminent en - $i$, - $e$, qui est la marque du cas Absolutif en hourrite. Par exemple : eše 'terre', bawurni 'ciel', sizwe, sizye 'eau', erbi 'chien', nali 'chevreuil', umini 'pays', ardi 'ville', Babani 'montagne', tahi 'homme', ašte 'femme', abi, ayi 'visage', edi 'corps', uruni 'cul, derrière', uri 'pied', šmmi 'main', etc. Quelques mots ont une finale $-a$, ce qui les trahit comme des emprunts : išna 'pluie' < akkadien zannatu.

- les termes de parenté. Ils se terminent normalement par -ai, mais il est fréquent que -ai se monophtongue en $-e,-i$. Par exemple, attai 'père', nera(i) 'mère', šen(na)i 'frère', elai 'soeur', $\beta u t k i$ 'fils', šalai 'fille', etc. Dans ces mots le $-i$ final est mobile et peut s'élider devant certains suffixes possessifs : senniwwe 'mon frère' mais šennab 'ton frère'. Il semble exister une certaine variation dialectale dans l'élision ou le maintien du $-i$ mobile.

- les théonymes. Certains se terminent par une consonne: Teššnb, Hebat, Kušuh, ce qui est exceptionnel en hourrite, ou par la voyelle - $a$, qui a parfois mais pas toujours une origine sémitique: Šauškea, Dakita, etc. Kumarbi, Aštabi ont une forme plus proche des noms ordinaires. Les théonymes se terminant pour une consonne se déclinent de façon particulièrement aléatoire. Cf. $\$ 9.13$.

- les noms propres. Le type de formation le plus répandu et le plus fréquent est la composition : Verbe+nom. Le verbe est souvent au Prétérit archaïque à finale $-b$, mais le $-b$ peut être omis. Exemples : Arib-Tě̌šnb, Pudu-Hebat, etc. D'autres formations sont possibles avec des adjectifs et d'autres temps que le Prétérit.

- les nombres. Ces lexèmes s'apparentent aux noms communs mais s'en distinguent par leur sens : šukki ' 1 ', sini '2', kig '3', tumni '4', neriya '5', etc. Certains nombres sémitiques sont également attestés : -, sini, -, irwi, himzat, etc.

9.2. Les différents types de suffixes se concatènent sur une base nominale dans un ordre prédéterminé :

- base nominale

- suffixe dérivationnel ( 0,1 ou plus) 




Une chaîne suffixale telle que ašbušikkeunniniwi qui comporte un article -ni-et une marque de cas -wi- peut à nouveau être suffixé par un article, ici -nna-, et une marque de cas, ici-ašta. Cf. \$2.8.

9.3. En règle générale, les bases nominales ont la forme $C \nu C$-, $C v n C-, C v / C-, C v n C-$ ou $v C$. Les groupes d'occlusives sont très rares: $\beta u t k i$ 'fils', itki 'sacré', Tupkiš. L'emprunt indo-iranien sapta

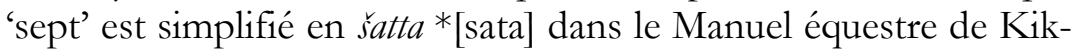
kuli. Le dialecte archaïque méridional subaréen semble plus tolérant que le hourrite mittannien vis-à-vis des groupes de consonnes : $\beta u t k i$ 'fils', Tupkiš sont anciens et subaréens.

Normalement l'initiale ne peut pas être sonore, ni être $r$ ou $l$. Les emprunts reçoivent des prothèses vocaliques : libittu $>$ alipši 'brique crue', Rašapu $>$ Eršep, akkadien zannatu $>$ išena $*$ [izena] 'pluie'. Les emphatiques akkadiennes sont adaptées en sonores : $q$, $s, t$, deviennent respectivement $g, z^{*}[\mathrm{~d} z]$ et $d$. Ce phénomène jette un doute sérieux sur les théories qui veulent que les emphatiques akkadiennes soient des sourdes glottalisées.

9.4. Les suffixes ont généralement la forme $-v C(v)$ - ou $-v C C(v)$-. La voyelle intercalaire des suffixes est généralement stable mais certains suffixes ont des variantes. Par exemple le suffixe d'Ergatif est le plus souvent $-i{ }^{2}$ : erbiniš 'le chien (Erg.)', mais Teššnb devient Teššbarš et Hebat Hebattuš. Pour cette raison, il est plus simple de classer les suffixes dans l'ordre alphabétique de leur (première) consonne.

9.5. Les suffixes dérivationnels les plus fréquents pour les bases nominales sont les suivants :

-vbi, -vwi : un suffixe possible bien que souvent la base nue ne soit pas attestée. Exemples : šerrewi 'agneau', karubi 'grenier, silo'. Erbi 'chien' est probablement un emprunt indo-iranien basé sur la racine *whp 'loup, renard'.

-(a)di: en général ce suffixe s'ajoute à des bases intransitives. Exemples : keldi 'bonne santé' < kel- 'être en forme', tumnadi 'quartette, quarteron' < tumni 'quatre'. Rien n'assûre que kumdi 'tour' repose sur un verbe non attesté *kum- 'construire'.

-aga, -akka: forme des diminutifs. Exemples: astakka 'jeune femme, jeune fille' < aste 'femme', tahakka, tahaga 'jeune homme' $<$ tabi 'homme'. On peut noter qu'une finale $-a$ ne présuppose pas un référent féminin. La déesse Šauška contient certainement une variante courte de ce suffixe. Cf. le suffixe $-u s i$.

$-(u) h i$ : forme des adjectifs ethniques. Normalement la voyelle intercalaire pour ce suffixe est $u$. Les mots sémitiques à finale $-a$ sont suffixés directement par - hi. Certains toponymes sont suffixés sans voyelle intercalaire, ce qui les signale comme étrangers à la langue hourrite. Exemples : Halbabi 'venant d'Alep, Aleppois' < 
Halba 'Alep', Tigrishi 'du Tigre' < Tigris 'le Tigre', Hattubi 'hittite, hatti' < Hatti 'pays hatti, hittite'.

-(u)hbi: forme des adjectifs qualificatifs. Exemples: turubhi 'masculin' < tabi 'homme', aštubhi 'féminin' < ašte 'femme'. Ce suffixe est proche du précédent.

$-i,-e:$ la marque d'Absolutif est aussi un suffixe à fonction nominalisante. Exemple : hani 'enfant' < han- 'enfanter'.

-mi : forme des dérivés de verbe (souvent à valeur passive). Exemples : elami 'serment [ce qui est dit]' < al- 'dire, crier', halmi 'chant', purami 'serviteur, esclave'.

-ni: (1) valeur particularisante. Exemples: sidari 'le fait de maudire' $\neq$ šidarni '(une) malédiction', dans d'autres cas la base suffixée diffère peu de la base nue : attai = attani 'père', hawur(ni) 'ciel', umini 'pays'. NB : ne pas confondre avec - ni 'Article Défini SG'.

$-n i,-u(n) n i$ : (2) dérivé verbal concret (actif), sert souvent à former des noms de professions. La base n'est pas toujours attestée à l'état nu. Exemples: Barini 'boulanger', Barandarini 'cuisinier', wudarini 'laveur de vaisselle', hašikeuni 'sourd' < baškeku 'il n'entend pas'. NB : ne pas confondre avec - ni 'Article Défini SG'.

-and-, -und-: forme des adjectifs. Exemples : abi 'visage, devant' $>$ awandalli 'antérieur', uruni 'derrière, cul' > urundalli 'postérieur'.

-ardi : forme des noms collectifs (animés). Exemples : šalardi 'ensemble des filles' < šali 'fille', attardi 'ancêtre(s)' < attai 'père', ardardi 'citoyen(s) (d'une ville)' < arde 'ville', elardi 'parentèle féminine' < eli 'sœur'.

-(u) si : intensif, augmentatif. Exemple : pedaruš 'grand taureau (au cas Abs.)', ne pas confondre avec pedaris' 'taureau (au cas Erg.)'. Cf. les suffixes -aga, -akka.

-(i) «̌sse/-(i) šsi: forme des dérivés abstraits à partir de noms (ou d'adjectifs). Exemple : šerišše, šeršse 'trone, royauté' < šäri 'roi'.

$-(v)^{5} k i$ : forme des dérivés, normalement sur base verbale. Exemples : tadaraški 'ami' < tad- 'aimer', summiluški 'apprenti' < šmmi 'main'.

9.6. Quelques suffixes longs s'apparentent plus à de la composition qu'à de la suffixation. Ils sont fréquemment utilisés avec des mots empruntés :

-dan, d'après le verbe tan- 'faire'. Endan 'prêtre', d'après eni 'dieu' (< sumérien AN 'ciel'), abuldanni 'portier', d'après akkadien abullu 'porte'.

- ubli 'en charge de, responsable de', d'après ebli 'voir, veiller à'. Nombreux exemples en relation avec des emprunts akkadiens. Akkadien eseédu 'récolte' > įadubli 'récolteur', akkadien halsu 'forteresse' > halzubli 'gouverneur', akkadien pilakku, pilaqqu 'quenouille' > pilakub(u)li 'fileuse', akkadien sagullu 'troupeau' > qugullubli 'pâtre, gardien de troupeau'.

-arbu 'âgé de $\mathrm{x}$ années': šinarbu 'âgé de deux ans', kigarbu 'âgé de trois ans', tumnarbu 'âgé de quatre ans', neriyarbu 'âgé de cinq ans', etc. 
9.7. Une base nominale, éventuellement augmentée de suffixes dérivationnels, peut recevoir des suffixes pronominaux possessifs. Ceux-ci ont éventuellement deux formes différentes, selon que la concaténation s'arrête à ce point ou se poursuit.

\begin{tabular}{|l|c|c|c|}
\hline & P1SG & P2SG & P3SG \\
\hline En fin de mot & $-w w e$ & $-b$ & $-a$ \\
\hline Enchaîné & $-w w w-$ & $-b e-$ & $-(y) i-$ \\
\hline
\end{tabular}

Suffixes pronominaux possessifs (Singulier)

Les suffixes du Pluriel sont partiellement déductibles des formes du Singulier par addition d'un suffixe $-a \grave{s}$.

\begin{tabular}{|c|c|c|c|}
\hline & P1PL & P2PL & P3PI \\
\hline En fin de mot & \multirow{2}{*}{$-w w a s$} & $-\check{s} s i$ & \multirow{2}{*}{$-y a s^{2}$} \\
\hline Enchaîné & & $-\check{s} \tilde{s} u-$ & \\
\hline
\end{tabular}

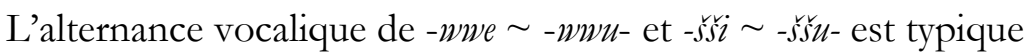
des pronoms. La voyelle finale de ces pronoms peut s'élider si le suffixe suivant est la conjonction enclitique -an 'et' ou bien un pronom enclitique mobile. Exemples :

(1) cas général d'un nom commun se terminant par -i, -e. Le suffixe possessif s'ajoute à la base, sauf pour P3SG où - $a$ remplace $-i,-e$ : umini 'pays': uminiwwe 'mon pays (Abs.)', uminiwnuwa 'à mon pays (Dat.)', uminib 'ton pays (Abs.)', umina 'son pays (Abs.)' umininwaš 'notre pays (Abs.)', uminiyaš 'leur pays (Abs.)', uminiyaša 'à leur pays (Dat.)'.

(2a) terme familial en -ai : le - $i$ final est théoriquement mobile [Cf. attani 'le père (Abs.)'] mais se maintient: attainwe 'mon père (Abs.)', attaibus' 'ton père (Erg.)', attaiwnaš 'notre père (Abs.)'.

(2b) terme familial en -ai: dans le cas du mot šenni 'frère', certaines formes reposent sur une base senna: šnniwwe 'mon frère (Abs.)', šennab 'ton frère (Abs.)', šenna 'son frère (Abs.)'.

Bien distinguer : eni 'dieu (Abs.)', enni 'le dieu (Abs.)', ena 'son dieu (Abs.)', enna 'les dieux (Abs.)', enniš 'le dieu (Erg.)', ennaš 'son dieu (Erg)', ennašuš 'les dieux (Erg)', eninnašuš 'ses dieux (Erg)'.

9.8. Après les suffixes dérivationnels et les suffixes pronominaux possessifs, un mot peut être suffixé par des Articles.

\begin{tabular}{|l|c|c|}
\hline & SG & Pl \\
\hline Abs. Indéfini & tabi & tabilla \\
\hline Abs. Défini & tabini & tabinna \\
\hline
\end{tabular}

Articles Définis et Indéfinis à l'Absolutif

Il faut noter que les suffixes -lla et -nna, qui fonctionnent ici comme articles, respectivement 'des' et 'les', peuvent aussi être 
des pronoms enclitiques mobiles (PEM). Normalement l'Article Défini au Pluriel est -nna: ebri 'seigneur (Abs.)', ebrinna 'les seigneurs (Abs.)'. Les marques de cas s'ajoutent aux articles - ni et nna. Le suffixe -lla est lui-même à l'Absolutif et ne peut pas recevoir de marque de cas.

Une base nominale dotée d'un suffixe possessif ne prend pas l'article défini au SG : *̌̌nniwwu-ni 'le mien frère' est impossible, mais * senniwnz-lla est possible : ici -lla est un PEM 'P3PL'. Enfin šnniwnu-nna 'mes frères' est sans doute possible mais non attesté.

Lorsque la dernière consonne de la base est $r$, l ou $n$, il se produit différentes complications phonétiques (assimilation, resyllabication): (1) nihari 'dot', niharri < *nihar(i)ni, (2) šuhni 'mur', šubunni $<*$ šuh(u)n(i)ni, (3) eli 'sœur', elli $<*$ el(i)ni. Dans les emprunts le $-m$ de la mimation sémitique et le $-m$ de l'accusatif indoiranien s'assimilent à l'article, d'où -nni.

9.9. Les suffixes de cas viennent ensuite. On peut distinguer deux types de suffixes : les cas grammaticaux, qui indiquent le rôle des mots dans la phrase et les cas locaux, qui se traduisent souvent par des prépositions en français. Les marques de cas sont largement stables et identiques pour les noms et les pronoms. Il n'existe en quelque sorte qu'une seule déclinaison.

Les principaux cas grammaticaux sont :

$-i,-e$ 'cas Absolutif. Exemples : tabi 'homme', tabini 'l'homme'. C'est la forme de citation au dictionnaire. La finale $-i,-e$ a une valeur nominalisante (Cf. \9.5). Le cas Absolutif est utilisé pour les sujets de verbes intransitifs ou passifs et les COD de verbes transitifs. Le cas Absolutif a aussi une valeur Locative.

-(i) $)^{2},-(e) s^{r}$ 'cas Ergatif. Exemple : tabinis 'l'homme (Erg.)'. C'est la forme prise par le sujet des verbes transitifs. Cette marque semble omise quand le verbe est un verbe de parole : 'dire', 'parler', etc. même lorsqu'un COD est présent dans la phrase. Le cas Ergatif peut aussi être utilisé comme complément de moyen dans une phrase où le prédicat est un adjectif ou un participe.

-wi, -we 'cas Génitif. Exemple : tabiniwi 'de l'homme'. C'est le cas des compléments du nom. Au pluriel ce suffixe est $-\check{s} i$, $-\grave{s}$, sauf à Nuzi qui présente la forme régulière $-5_{\text {šmi }}$ ou $-\check{s} w$ we.

-wa 'cas Datif. Exemple : tahiniwa 'à l'homme'. Au pluriel ce suffixe est $-(v) s_{a} a$ sauf à Nuzi qui présente la forme régulière $-s_{w} w a$. Ce cas a deux fonctions: le destinataire et le complément des noms positionnels : tabiniwa abi 'devant l'homme', litt. 'en face à l'homme'.

-nna 'cas Equatif. Exemple : tabinna 'en tant qu'homme'. Ce cas peut aussi s'analyser comme étant un adjectif dérivé tabinn'humain, viril' suivi d'une marque prédicative $-a$.

Les principaux cas locaux sont :

-da 'cas Allatif. Exemples : ardinida 'vers la ville', au Pluriel ardinnašta. Indique la direction d'un déplacement. Ce cas semble pouvoir aussi exprimer le Datif. 
-dan 'cas Ablatif. Exemple : ardinidan 'hors de, venant de la ville', au Pluriel ardinnaštan. Exprime la provenance. NB: la matière est exprimée par le suffixe -uhbi: sinniberubhi 'en ivoire'.

- $a$ 'cas Locatif. Exemple : hawurni(y)a 'dans le ciel'.

$-i$ 'cas Locatif. Ressemble à l'Absolutif utilisé sans marque de cas supplémentaire. Exemple : epheni 'dans le four'.

- ae 'cas Instrumental'. Exemple : urae 'avec le(s) pied(s)'. Ce cas semble neutraliser l'opposition Singulier $\sim$ Pluriel.

-ura 'cas Comitatif. Exemple : tahinnašura 'avec les hommes'.

Les cas autres que les cas Absolutif et Ergatif sont obliques.

9.10. Le Pluriel est exprimé par -lla à l'ABs. indéterminé et par le suffixe prédésinentiel $-\check{s}$ dans les autres cas :

\begin{tabular}{|c|c|c|}
\hline & SG & PL \\
\hline Absolutif & $-\varnothing,-i,-e$ & $-1 l a$ \\
\hline Ergatif & $-\check{s}$ & $-s^{2} u s^{2}$ \\
\hline Génitif & $-w e,-w i$ & $-{ }^{r}[w] e,-\bar{s}[w] i$ \\
\hline Datif & $-w a$ & $-s[w]] a$ \\
\hline Locatif & $-a,-i$ & $-s^{2} a$ \\
\hline Allatif & $-d a$ & $-s ̌ c t a$ \\
\hline Ablatif & $-d a n$ & $-s \tan$ \\
\hline Comitatif & $-r a$ & $-{ }^{2}$ sura \\
\hline Equatif & $-n n a$ & -šnnna \\
\hline
\end{tabular}

Combinaison des marques de cas et du Pluriel

Ces suffixes sont valides pour toutes les bases nominales et pronominales, et les dépendances syntaxiques (adjectifs, etc.).

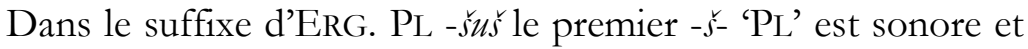
s'écrit avec une simple, mais le deuxième qui marque l'ERG. est sourd et s'écrit avec une géminée graphique - $-\mathfrak{s}$ š- en cas de suffixation par un PEM, par exemple -a(nna) 'le, la' ou une ENC, par exemple -an 'et'.

9.11. Normalement les suffixes de cas s'ajoutent aux bases au moyen d'une voyelle intercalaire. Lorsque le nom est suffixé par l'article $-n i,-i$ sert de voyelle intercalaire et les suffixes de cas peuvent s'ajouter directement, mais à l'INST. -ni-ae devient -nae, et au Com. -ni-ura devient -nura. Avec l'article Pluriel -nna, -a sert de voyelle intercalaire et les suffixes de cas peuvent s'ajouter directement, mais l'ERG. est -nnašus.

Les pronoms et déictiques remplacent la finale $-i,-e$ de l'ABS. par $-u$, auquel s'ajoutent les suffixes de cas obliques, mais l'ERG. est $-i \Sigma^{2}$ ou $-e \check{r}^{\circ}$. Cette alternance $\mathrm{i} / \mathrm{u}$ est typique des pronoms.

Dans les noms se terminant par $-a$, comme par exemple Šauška, cette voyelle se maintient à tous les cas: ERG. Šauškaš, GEN. Šauskeawi, etc. 
9.12. Les dépendances d'une base nominale - adjectifs, formes verbales suffixées par le relatif $r e$ - reçoivent les mêmes articles et marques de cas que celle-ci. Il y a donc accord formel.

- ulbi-ni paban-ni 'l'autre montagne (ABS. DEF)'

- niharrivi 'de la dot (GEN. DEF)', niharriwi arušauseniwi 'de la dot, que j[e l]'ai donnée (GEN. DEF)' où arušauše est une forme verbale arušau 'je l'ai donné(e)', nominalisée par le relatif -še. Une fois nominalisée cette forme peut recevoir des articles et marques de cas, qui précisent à quel mot elle se rapporte.

9.13. Les théonymes se terminant par une consonne doivent être appris individuellement :

(1) Tešsub a pour ERG. Tě̌šubaš, Gen. Tešsuppi, DAT. Teššppa, et la base oblique est Teřšsuba- pour les autres cas.

(2) Hebat a pour ERG. Hebattus, GEN. Hebatti ou Hebatt(e)wi, les autres cas reposent sur Hebatte-.

(3) Kứsub a pour ERG. Kušubišs, GEN. Kušsubhi, DAt. Kušsubha, et pour base oblique $K u \dot{s} u h u$ - pour les autres cas.

(4) Anat a pour base suffixée Anata-.

Certains théonymes sont utilisés avec l'article défini -ni. Cela indique qu'ils sont perçus comme étrangers ou composés : EaŠärri-ni, Pišašaphi-ni, etc. Le théonyme Kumarbi reçoit parfois l'article : Kumarbi-ni, mais il est généralement nu. Il est à mon avis impossible que ce mot soit un Génitif, comme cela est parfois proposé, car on ne voit pas comment les locuteurs pourraient utiliser une forme de Génitif avec un sens Absolutif, voire pire lui rajouter un suffixe d'Ergatif. Il est possible que ce soit un composé kum-arbi 'âgé de kum [100?] années' (Cf. §9.6.). Cela expliquerait qu'il puisse recevoir l'article défini. Le théonyme Šmegi est semble-t-il toujours utilisé avec l'article.

Normalement les théonymes sont précédés du signe DINGIR 'dieu', dans les textes cunéiformes.

9.14. Quelques formes nominales ont une finale $-u$. Elles ont normalement une valeur positionnelle: telu 'en haut', tapš́ 'en bas', utburu 'sur le côté'. D'autres sont plus adverbiales : kulu 'à nouveau', inu 'combien, comment'. Certaines conjonctions ont cette finale : panu- 'bien que', undu- 'alors, au moment où'. Le suffixe -arbu 'âgé de $\mathrm{x}$ années' a aussi cette finale $-u$.

9.15. Les nombres s'apparentent aux formes nominales. Ils peuvent recevoir les mêmes marques de cas : tumni-lla 'les quatre'. Du fait de leur sens, les possibilités de suffixation sont réduites. Les principaux suffixes dérivationnels pour les nombres sont:

$-d i$ : tumnadi 'quartette, quarteron' < tumni 'quatre', emandubli 'décurion' < eman 'dix'.

$-(\tilde{s}) \tilde{s i},-(\tilde{s}) \breve{s e}$ : forme les ordinaux, kikšse, kiške (avec métathèse) 'troisième' < kig 'trois'. Il peut aussi se suffixer aux nombres sémitiques : imvišse traduit le mot akkadien ilqu 'impôt' et signifie litt. 'quatrième'. 


\section{La morphologie verbale}

10.1. La morphologie verbale est complexe du fait du nombre de paramètres intervenant dans l'analyse en signifiés élémentaires : pronoms personnels Sujet ou COD, diathèses, temps, modes, forme affirmative ou négative. A cela s'ajoutent des variantes et des archaïsmes. La documentation disponible n'atteste pas toutes les formes que le système laisse entrevoir comme possibles. La morphologie verbale doit être apprise en même temps que les pronoms (Cf. \$12), où elle puise une bonne partie de ses suffixes formatifs.

Les bases verbales sont soumises aux mêmes contraintes phonotactiques que les noms (Cf. \$9.3).

La conjugaison est organisée en trois diathèses de base, qui sont parallèles à l'opposition entre les cas Absolutif et Ergatif :

- verbes de mouvement, d'état, adjectifs, à un actant (à l'ABS.)

- verbes intransitifs, à un actant (à l'ABs.)

- verbes transitifs, à deux actants (à l'ABS. et à l'ERG.).

A la $3^{\text {ème }}$ personne il y a donc trois jeux de suffixes :

- diathèse1 : una 'il vient', avec finale - $a$.

- diathèse1 : mana 'il est', avec finale - $a$.

- diathèse1 : Bura 'il est fort', avec finale - $a$.

- diathèse 2 : anu 'il se réjouit', avec finale - $u$.

- diathèse 3 : tadia 'il ou elle l'aime', avec finale -ia.

Les verbes de mouvement, normalement intransitifs, peuvent être mis à la diathèse transitive, avec le changement de sens qui convient au mouvement en question :

- diathèse1 : una 'il vient', avec finale - $a$.

- diathèse 3 : unia 'il ou elle l'apporte', avec finale -ia.

Chaque diathèse a sa propre expression de la négation:

- diathèse1 : unukku 'il ne vient pas', avec finale -ukk-u.

- diathèse 2 : anikk ki 'il ne se réjouit pas', avec finale -ikk-i.

- diathèse 3 : tadia(m)ma 'il ou elle ne l'aime pas', avec une finale -ia(m)ma, combinant -ia et -(m)ma. La NEG dépend de la personne et du temps pour cette diathèse 3 .

Concernant mana 'il est', outre manukku 'il n'est pas', il existe une forme archaïque manubur dont l'analyse en morphèmes n'est pas claire, bien que -ub- semble indiquer la négation.

10.2. Le mode Indicatif a 3 temps: Présent, Prétérit, Futur. Puis viennent les modes Conditionnel, Optatif et Désidératif qui n'existent qu'au Présent. L'Impératif n'existe qu'au Présent.

Il existe un infinitif de type nom d'action en -umma, et un jeu complexe de participes, qui sont tantôt des formes nominales, tantôt des formes adjectivales. Cf. \$10.12. et \$11.7. 
10.3. Comme avec les noms, les suffixes se concatènent sur une base verbale dans un ordre prédéterminé. On reconnait deux types de chaines suivant que le verbe implique un seul actant ou deux actants.

Chaîne à un seul actant :

- base verbale

- suffixes dérivationnels ( 0,1 ou plus)

- marque de temps ou mode (1 seule)

- négation (le cas échéant)

- pronom enclitique mobile PEM

Chaîne à deux actants :

- base verbale

- suffixes dérivationnels $(0,1$ ou plus $)$

- marque de temps ou mode (1 seule)

- suffixe pronominal actif (1 seul)

- négation (le cas échéant)

- pluriel

- pronom enclitique mobile PEM

Les deux types de chaînes diffèrent donc par l'insertion après la marque de temps ou de mode d'un suffixe pronominal actif. Sinon l'ordre des éléments est sensiblement le même.

Les PEM sont implicitement au cas ABS. Ils correspondent donc au sujet dans la chaîne intransitive à un actant et au COD dans la chaîne transitive à deux actants. Les suffixes pronominaux actifs sont implicitement à l'ERG. et expriment donc le sujet d'un verbe transitif.

Normalement la concaténation est transparente et analytique, mais il existe quelques cas de suffixes pronominaux actifs plus ou moins fusionnés avec la négation, en une sorte d'amalgame. Dans une large mesure la morphologie ressemble à une sorte de mécano morphémique où une bonne partie des formes est presque prévisible. Toutes les formes ne sont pas attestées et ne permettent pas de vérifier que ce mécano existe effectivement.

10.4. Les PEM peuvent être suffixés à un autre élément que le verbe, ce qui peut faussement suggérer que le verbe est à la P3SG.

- una 'il ou elle vient'

- unadilla 'nous venons'

- bennidilla una 'nous venons maintenant'

Le PEM -dilla 'P1PL ABs.', bien que suffixé à l'adverbe henni 'maintenant', est le sujet réel du verbe un-. La suffixation déportée des PEM est un phénomène typique du hourrite, qui peut être une source de confusion : una a l'apparence suffixale d'une 'P3SG' mais le PEM -dilla présent sur un autre élément de la phrase indique quelle est la vraie personne du verbe : ici, 'P1PL'.

10.5. Les suffixes dérivationnels les plus fréquents pour les bases verbales sont les suivants :

-ad- : (sumérien) SÌR 'chant'> sirad- 'chanter' 
-ud- 'valeur inversive (?) in-, ré-' (pas de variante -id-, -ad-) : ₹ulud- 'dénouer', [Proposé par M. Giorgieri, mais controversé]

-(u)gar-'duel, réciproquement': tad- > tadugar- 's'aimer l'un l'autre', ugulgar- 's'agenouiller, plier les (deux) genoux'

-am-'factitif' : eman 'dix'> emanam- 'décupler'

- ah(b)- 'causatif -iser, -ifier' (pas de -ihb-, -ubh-) : tal- 'élevé, haut' $>$ talah- 'enlever, prendre', mel- 'loin (?)' > melah- 'chasser, expulser'

-uh- 'sens peu clair' : his- (akkadien) > hisub- 'être en colère'

-ib- 'sens peu clair' : *ananibi 'menthe', pusû (akkadien) > puzih'nettoyer, plonger dans l'eau'

-il- : al- 'parler, prêter serment' > alilan- 'crier, se lamenter', ulil'mourir' ulmi 'arme'

-ill-'inchoatif [se mettre à]' : sidarill- 'se mettre à maudire à maintes reprises'

-ul- '(?) parfois médio-passif' : agul- 'sculpter', arul- 'guider, mener', arušsul- 'se dépêcher'

-al-: itku 'sacré' > itkal- 'purifier, consacrer', hešal- 'être nu' (?) hišmi 'clair' < *biš- 'être visible (?)'

-an- 'parfois causatif' (pas de variante -in-, -un-) : al- 'parler, prêter serment' > alilan- 'crier, se lamenter', hab- '(?)' > haban- 'se déplacer, se rendre à', ki- 'placer' > kiban- 'apporter', uett- 'avoir faim' > ulan- 'manger'

-ang- 'sens peu clair' (pas de variante -ing-, -ung-) : pud- > pudang'annoncer'

-ar- 'normalement itératif (variante -ir-, -ur-) : šid- 'maudire' > šidar- 'maudire à maintes reprises', -ur- : aguri 'sculpture', -ir- : adirha 'étant en conflit'

$-s t$ - 'intensif, ce suffixe est harmonique et prend la voyelle qui précède : an- $>$ anašt- 'se réjouir', bub- 'casser' $>$ bubust- 'réduire en miettes', zamalašt- 'détruire, déchirer'

- ̌́- 'intensif' : hab- '(?)' > bap̌̌ar- 'marcher en permanence', haš'entendre' > hašaš- 'entendre bien', tap- 'bas (?)' > tapšs, tašp'mettre à bas, détruire' 'aimer'

-imb- 'sens peu clair' ${ }^{32},-u p p$ - 'sens peu clair': tad- > tad-upp-

10.6. Principaux dérivés nominaux de bases verbales :

- $m i$ 'dérivé normalement passif sur base transitive $=$ ce que, celui que' : halmi 'chanson'

$-n i$ 'dérivé normalement actif sur base transitive $=$ ce qui, celui qui' : wudarini 'laveur de vaisselle'

- di 'normalement sur base intransitive' : keldi 'bonne santé'

-išhi 'normalement causatif actif $=$ celui, ce qui fait faire' : paššišbi 'envoyeur, commanditaire'

-ithi 'normalement causatif passif = celui, ce qui est fait faire' : pašsitthi 'envoyé, embassadeur'

32 Dietrich - MAYER, op. cit. (n. 15), p. 159, proposent un sens intensifduratif pour ce suffixe. 
-umma 'infinitif : ašbi 'haut' > ašbulumma 'élévation, sacrifice'

10.7. Les marques de temps et modes sont les suivantes :

- INDI PRES : non marqué, $\varnothing$

- INDI PRET : marqué par -uš-

- INDI FUT : marqué par -ed-, ou en variante par -id-

- COND : marqué par -(e)wa-, -(i)wa-

- OPTA : marqué par -e-, ou en variante par - $i-$

- DESI : marqué par -el-, ou en variante par -il-

Le suffixe $-u \check{s}$ - est omis dans les formes de Prétérit archaïque : unab 'il est venu, ils sont venus' et non pas **unušab, de même tandib 'il fit, ils firent' et non pas **tandušib. La finale $-b$ n'est pas compatible avec la marque de temps $-u \check{s}$. Les formes archaïques en - $v b$, qui étaient syncrétiques pour 'P3SG' et 'P3PL', ont été remplacées au $2^{\text {ème }}$ millénaire av. J.-C. par des formes analytiques en $-u s_{a} a$ et $-u s ̌ t u$. Les formes archaïques à finale $-v b$ ont néanmoins perduré pendant quelque temps - quelques siècles - dans l'onomastique, après quoi le $-b$ final s'est amuï définitivement: AribTeššnb 'Teššub l'a donné(e)' aboutit au diminutif $A r$-Teya.

Le Conditionnel est le mode de la irréalité, l'Optatif celui des actions espérées ou souhaitées, le Désidératif concerne les actions désirées par celui ou celle qui s'exprime.

En apparence le Futur et le Désidératif semblent être dérivés de l'Optatif $-\ell-$ par des suffixes divergents : $-e d-$, $-e l$. C'est peutêtre aussi le cas du Conditionnel. A la forme négative le suffixe du Désidératif est-ewall-, et le morphème -wa- sépare - - - de - $l-$. Cette situation renforce la présomption que le Désidératif a une origine secondaire et suffixale. L'Optatif semble être un temps ancien dans la langue et les suffixes pronominaux sont assez divergents et atypiques, en comparaison de ceux de l'Indicatif. Cf. \$12.6.

Il est possible que le suffixe - - - de l'Optatif soit un ancien *aya monophtongué. C'est ce que tend à montrer la forme tapšihayalli du Lion d'Urkeš, qui est un Désidératif HCN tapšiheli(n).

10.8. L'Impératif se forme de la façon suivante:

Verbes de mouvement: una 'viens!'

Verbes intransitifs : Kelu 'porte-toi bien!'

Verbes transitifs : ari 'donne(-le)!'

Les formes du type una et kelu (Diathèses1 et 2) peuvent être suffixés par le PEM 'P2SG' court - $m$ ou long -mma, en fonction de sujet intransitif explicite : unam(ma), kelum(ma).

Les formes du Pluriel sont :

Verbes de mouvement: unap $(p a)$ 'venez!'

Verbes intransitifs : kelup $(p a)$ 'portez-vous bien!'



Pour 'P2PL' le PEM court - $p$ ou long - $p$ pa est obligatoire, car il exprime aussi le Pluriel. Ces formes d'Impératif sont identiques à celles de l'Indicatif Présent: unap(pa) 'vous venez', kelup(pa) 'vous 
vous portez bien', $\operatorname{aris}(\check{s} u)$ 'vous (le/la) donnez'. C'est donc le contexte d'énonciation qui fait la différence.

10.9. L'opposition de deux diathèses intransitive et transitive se traduit par un double jeu de suffixes pronominaux. Par exemple au Présent de l'Indicatif:

- 'P1SG' un-a-tta 'je viens'

- 'P2SG' un-a-mma 'tu viens'

$\neq \quad$ tad-au 'je l'aime'

- 'P3SG' un-a 'il vient'

$\neq$ tad-iu 'tu l'aimes'

- 'P1PL' un-a-dilla 'nous venons' $\neq$

- 'P2PL' un-a-ppa 'vous venez'

- 'P3PL' un-a-lla 'ils viennent'

$\neq$ tad-ia 'il/elle l'aime'

$\neq \operatorname{tad}-a u s(a)$ 'nous l'aimons'

$\neq \quad$ tad-ašsu 'nous l'aimons'

$\neq$ tad-idu 'ils/elles l'aiment'

Pour obtenir les formes verbales transitives avec un autre COD que 'P3SG' il faut suffixer le PEM concerné: 'P1SG'-(a)t(ta), 'P2SG' - (a)m(ma), 'P1PL' -(a)dil(la), 'P2PL' - (a)p(pa), 'P3PL' - (a)l(la).

Les formes intransitives à l'Indicatif sont en théorie :

- 'P1SG' PRES un-a-tta $>$ FUT un-ed-a-tta, PRET un-uš-a-tta

- 'P2SG' PRES un-a-mma > FUT un-ed-a-mma, PRET un-uš-a-mma

- 'P3SG' PRES un-a $>$ FUT un-ed-a, PRET un-urs-a (Arch. unab)

- 'P1PL' PRES un-a-dilla > FUT un-ed-a-dilla, PRET un-uś-a-dilla

- 'P2PL' PRES un-a-ppa > FUT un-ed-a-ppa, PRET un-uš-a-ppa

- 'P3PL' PRES un-a-lla > FUT un-ed-a-lla, PRET un-uš-a-lla

Pour obtenir les formes négatives, il suffit d'insérer le segment -ukk-(a)-, sauf pour 'P3SG' PRES un-a qui fait unukku. La forme négative du PRET archaïque unab n'est pas connue.

Les formes transitives à l'Indicatif sont en théorie :

- 'P1SG' PRES tad-au > FUT tad-ed-au, PRET tad-uš-au

- 'P2SG' PRES tad-iu > FUT tad-ed-(i)u, PRET tad-ušu

- 'P3SG' PRES tad-ia > FUT tad-ed-(i)a, PRET tad-uš-a

- 'P1PL' PRES tad-auš(a) > FUT tad-ed-auš(a), PRET tad-uš-auš(a)

- 'P2PL' PRES tad-ǎšn > FUT tad-ed-ašsu, PRET tad-uš-ašsu

- 'P3PL' PRES tad-idu > FUT tad-ed-(id)u, PRET tad-uš-(i)du

Les $3^{\text {èmes }}$ personnes au Futur semblent syncrétiques : tadedu, tadettu ou tadeda, tadetta ne s'opposent pas clairement entre le SG et le PL. La suite des formes est étudiée avec les pronoms (\$12.5.).

10.10. Exemples de chaînes à 1 ou 2 actants :

- u-na-wz-at-ta 'je viendrais'

un- 'venir'

- suffixes dérivationnels $(=\varnothing)$

- marque de temps ou mode $(=-a-w a-)$

- négation $(=\varnothing)$

$-\operatorname{PEM}(=-(a) t t a)$

- ku-zu-u-si-wo-wo-la (Mitt. IV 46) 'je ne les ai pas gardés'

kuz- 'garder, retenir'

- suffixes dérivationnels $(=\varnothing)$

- marque de temps ou mode $\left(=-u s^{-}\right)$

- suffixe de pronom personnel $(=-i w[u]-)$ 


$$
\begin{gathered}
\text { - négation }(=-w a-) \\
\text { - pluriel }(=\varnothing) \\
\text { - PEM }(=-(a) \text { lla })
\end{gathered}
$$

- na-ak-ki-u-ub-wว-uš (KBo 3215 I 24) 'nous ne le libérons pas' nakke- 'libérer, émanciper'

- suffixes dérivationnels $(=\varnothing)$

- marque de temps ou mode $(=\varnothing)$

- suffixe de pronom personnel $(=-i w[u]-)$

- négation $(=-w a-)$

$$
\begin{array}{r}
\text { - pluriel }(=-u \check{s}) \\
\text { - PEM }(=\varnothing)
\end{array}
$$

10.11. La NEG verbale s'exprime de différentes façons :

\begin{tabular}{|l|c|c|c|}
\hline & Diathèse1 & Diathèse2 & Diathèse3 \\
\hline INDI PRES & $-u k k-$ & $-i k k-$ & $\$ 12.5$. \\
\hline INDI PRET & $-u k k-$ & $-i k k-$ & $\$ 12.5$. \\
\hline INDI FUT & $-u k k-$ & $-i k k-$ & $\$ 12.5$. \\
\hline COND & $?$ & $?$ & $?$ \\
\hline OPTA & $-w e-$ & $-w e-$ & $-w e-$ \\
\hline DESI & $-w a l l-$ & $-w a l l-$ & $-w a l l-$ \\
\hline
\end{tabular}

Expression de la négation verbale

Le COND Neg ne semble pas attesté dans la documentation existante. La forme négative de l'Impératif, si tant est qu'elle existe, n'est pas connue.

10.12. Outre les formes conjuguées, il existe des formations de participes, combinant nature verbale avec morphologie nominale ou adjectivale. On peut identifier :

- PART Actif PRES : forme courte : -im (TRA), -um (INT), forme longue -ima(e) (TRA), -uma(e) (INT), avec marque de cas -a(e) INST. Exemples : hašimma 'entendant cela', alilanum 'en geignant', kunzimae 'étant agenouillé'

- PART Actif Passé : -iri. Exemples : pairi 'ayant construit', sizyaliri 'ayant placé', tabiri 'ayant fait couler (du métal)'

NB: Cf. tabiri 'forgeron'.

- PART Passif PRES : -adu. Exemples : binzadu 'étant oppressé, contraint', nabbibadu 'inhabité, désert' : lit. 'où personne n'est installé, assis'

- PART Passif Passé : -iliya. Exemples : pailiya 'qui a été construit', tabiliya 'qui a coulé, fondu', šeduiliya 'qui a été engraissé'

NB: L'exemple nabbibadu 'inhabité, désert' contient un segment -ib-de NEG verbale. 


\section{Les adjectifs}

11.1. Les adjectifs ont un fonctionnement hybride, qui les place à mi-chemin entre les noms et les verbes. Avec les noms ils partagent de recevoir les articles et les marques de cas; avec les verbes ils partagent d'être sensibles aux temps et aux modes. Ils peuvent en outre être suffixés avec la NEG verbale -ukk.

11.2. Il y a peu d'adjectifs primaires qui ne soient pas dérivés de noms ou de verbes : adal(i) 'fort', kalgi 'faible', muši 'juste', niri 'bon', tagi 'beau', talmi, telami 'élevé', timäri 'sombre', ubi 'stupide', ulbi 'autre', urbi 'vrai'.

La terminaison des adjectifs est variable $: i, e, u$ voire $\varnothing$.

11.3. On peut dériver un adjectif d'un nom ou d'un verbe avec le suffixe -nni: mad- 'être sage, raisonner' > madunni 'sage', šenni 'frère' > šennanni 'fraternel'.

Une autre formation est - $n d: a b i$ 'visage' > awandalli 'antérieur', uruni 'cul' > urundalli 'postérieur'.

11.4. Les principaux suffixes dérivationnels sont :

$-\stackrel{s}{s i} i,-\check{s} s e$ : forme des dérivés abstraits, niri ‘bon' > nirišse 'bonté'

-uši : intensif : talmi, telami 'élevé' > talawnsi, talbuš 'gigantesque'

-ae: la marque d'INST. forme des adverbes du type -ment: šenni 'frère' > šennanni 'fraternel' > sennannae 'fraternellement'

11.5. Les adjectifs peuvent être prédicat et attribut du sujet sans copule. Dans ce cas il arrive qu'ils prennent la marque $-a$, des verbes de mouvement et d'état. Ces formes sont principalement attestées dans l'onomastique :

- Enna-muša 'les dieux sont justes' (ordre des mots sémitique)

- Erbi-urbi 'le seigneur est vérité' (ordre des mots sémitique)

- Urbi-Teššnb 'Teššub est vérité'

- Talmi-Šarruma 'Šarruma est grand'

- Ebri-Talma 'Le seigneur est grand' (ordre des mots sémitique)

11.6. Les adjectifs peuvent recevoir les marques de temps et de modes. Les formes attestées sont incomplètes et ne donnent qu'une vision parcellaire des paradigmes sans doute possibles.

- Urbal-Enni 'Que le dieu soit vérité' (mode DESI)

On peut noter que dans urbal le suffixe de DESI n'est pas précédé par la voyelle $e$ de l'OPTA.

11.7. Il est possible que n'importe quelle forme verbale puisse être convertie en adjectif avec le suffixe -(a)nni. Cela expliquerait des formes telles que amelanni qui semble reposer sur le DESI du verbe am- 'brûler, incendier'. Cette sorte de participe peut avoir un sujet à l'ABs. et un complément de moyen ou de cause à l'ERG. On peut donc décrire le même évènement ou processus de façon active ou passive : 
- (forme passive) pabanni amelanni tarriš 'que la montagne soit incendiée par le feu !'

- (forme active) pabanni ameli(n) tarriš 'que le feu incendie la montagne!'

Les deux phrases reposent sur am- 'incendier' et -el- 'mode désidératif, avec un suffixe d'adjectif ou de participe -anni ou un suffixe d'Optatif $-i(n)$. Du fait de la structure ergative du hourrite le patient et l'agent ne changent pas de forme.

\section{Les pronoms personnels}

12.1. On peut distinguer cinq types de formes en relation avec l'expression des pronoms personnels :

- les pronoms libres indépendants, PLI : ils fonctionnent comme des noms mais leur fonction et leur sens est d'exprimer les pronoms personnels. Ils reçoivent les marques de cas. Ils équivalent à la série : moi, toi, lui, elle, nous, vous, eux, elles.

- les pronoms enclitiques mobiles, PEM : ils ne peuvent que se suffixer à un autre mot de n'importe quelle classe. Ils sont au cas ABS. implicitement et par conséquent ils ne sont pas déclinables. Ils expriment soit le sujet d'un verbe intransitif à 1 seul actant soit l'objet d'un verbe transitif à 2 actants.

- les suffixes possessifs, Poss : ils se suffixent à des noms, ou à leurs dépendances (adjectifs, verbes nominalisés, etc.). Ils ne se déclinent pas. Ils équivalent à mon, ma, mes, etc. mais ne sont pas des mots indépendants ou des déterminants comme en français.

- les suffixes pronominaux actifs de l'Indicatif, SPAI : ils se suffixent à des bases verbales. Ils sont implicitement à l'ERG. et expriment le sujet d'un verbe transitif. A la forme négative, il arrive qu'ils soient plus ou moins amalgamés avec l'expression de la négation verbale.

- les suffixes pronominaux de l'Optatif et du Désidératif, SPOD : ils se suffixent à des bases verbales. Ils divergent des SPAI et doivent être décrits séparément.

12.2. Les pronoms libres indépendants, PLI, présentent six jeux de formes pour les six personnes. Les formes du Pluriel sont en partie déductibles de celles du Singulier, mais 'P1PL' est la plus irrégulière de ce point de vue. Pour obtenir l'ensemble des formes, il suffit de connaitre l'ABS., l'ERG. et la base oblique, à laquelle s'ajoutent les suffixes de cas listés aux $\$ 9.9$. et $\$ 9.10$.

\begin{tabular}{|l|c|c|c|}
\hline & P1SG & P2SG & P3SG \\
\hline Absolutif & $i s t e$ & $\beta e$ & mane \\
\hline Ergatif & $i \check{s} a \check{s}$ & $\beta e \check{s}$ & manu $\check{s}^{\check{L}}$ \\
\hline Base oblique & $\check{s} u-$ & $\beta e-$ & manu- \\
\hline \multicolumn{2}{|c|}{ Pronoms libres indépendants PLI (Singulier) }
\end{tabular}




\begin{tabular}{|c|c|c|c|}
\hline & P1PL & P2PL & P3PL \\
\hline Absolutif & šattil(la) & $\beta e l(l a)$ & manel(la) \\
\hline Ergatif & sies & $\beta e \check{s} u \check{s}$ & manšuš \\
\hline Base oblique & $\check{s} a-$ & $\beta e-$ & manšu- \\
\hline
\end{tabular}

NB: Le symbole $\beta$ note l'alternance $w, p, b$ des graphies.

NB: Pour le pluriel aux cas obliques, prendre les suffixes $-\check{r}$-xx de la colonne de droite du $\$ 9.10$. Toutes les formes ne sont pas attestées mais elles semblent régulières.

12.3. Les pronoms enclitiques mobiles, PEM : ils constituent une particularité du hourrite. A noter spécialement :

- Ils peuvent se suffixer à peu près à n'importe quel mot de la phrase, éventuellement de façon pléonastique plusieurs fois.

- Un PEM, sujet d'un verbe intransitif, peut être suffixé à un autre élément que le verbe. Cf. $\$ 10.4$.

- Lorsqu'un nom est suffixé par un PEM, la marque d'ERG. est supprimée, puisque normalement le premier nom est sujet et le PEM est objet du verbe. La marque d'ERG. est alors redondante.

Les PEM ont deux formes, courte et longue. La forme courte s'obtient par suppression de la syllable $-C a$. Les formes longues sont : P1SG -tta, P2SG -mma, P1PL - dilla, P2PL - ppa, P3PL -lla. Le PEM P3SG -nna sert de pronom anaphorique.

Normalement les PEM sont précédés de la voyelle intercalaire $a$, ce qui permet de distinguer -a-lla PEM de $u / i$-lla PLURIEL.

12.4. Les pronoms Poss sont les suivants. Ceux-ci ont éventuellement deux formes différentes, selon que la concaténa-tion s'arrête à ce point ou se poursuit.

\begin{tabular}{|l|c|c|c|}
\hline & P1SG & P2SG & P3SG \\
\hline En fin de mot & $-w w e$ & $-b$ & $-a$ \\
\hline Enchaîné & - wwu- & $-b e-$ & $-(y) i-$ \\
\hline
\end{tabular}

Suffixes pronominaux possessifs (Singulier)

Les suffixes du Pluriel sont partiellement déductibles des formes du Singulier par addition d'un suffixe $-a \grave{s}$.

\begin{tabular}{|c|c|c|c|}
\hline & P1PL & P2PL & P3PL \\
\hline En fin de mot & \multirow{2}{*}{$-w w a s ̌$} & $-e_{s}^{-s i}$ & \multirow{2}{*}{$-y a s$} \\
\hline Enchaîné & & $-\check{s} \tilde{s} u-$ & \\
\hline
\end{tabular}

Voir aussi $\$ 9.7$. 
12.5. Les suffixes pronominaux actifs de l'Indicatif, SPAI :

\begin{tabular}{|c|c|c|}
\hline & SG & PL \\
\hline P1 & -au / -iunwe & $-a u s(a) /-i u m w u s(a)$ \\
\hline P2 & $-(i) u /$ ? & $-a \check{s} \tilde{s} u /-i u \check{s}(\check{s u} u)$ \\
\hline P3 & $-(i) a /-(i) a(m) m a$ & $-i d u$ / ? \\
\hline
\end{tabular}

SPAI à la forme affirmative et négative

Ces suffixes s'attachent aux bases verbales, par exemple tan- ou sidar-, ou aux marques de temps : $\varnothing, e d, u \check{s}$. Ensuite peuvent venir éventuellement des PEM, en fonction de COD.

12.6. Les suffixes pronominaux de l'Optatif et du Désidératif, SPOD, ne sont pas tous attestés. Ils sont conventionnellement écrits avec la voyelle $-e$ - mais on trouve aussi $-i-$. A la forme affirmative :

\begin{tabular}{|c|c|c|}
\hline & SG & PL \\
\hline P1 & $-i /-e l-i$ & $-i \check{s} /$-el-iwaš \\
\hline P2 & $? /-e l-a b$ & $?$ \\
\hline P3 & $-e n,-i n / ?$ & -den, -din / ? \\
\hline
\end{tabular}

SPOD à la forme affirmative

A la forme négative, les différents morphèmes se combinent dans un ordre atypique. La NEG semble être - wa? - et l'arrêt glottal provoque soit un hiatus soit une géminée -wall- au DESI :

\begin{tabular}{|c|c|c|}
\hline & SG & PL \\
\hline P1 & $-i /$-iwall-i & $?$ \\
\hline P2 & $?$ & $?$ \\
\hline P3 & -iwa-en, / ? & -idu-w-en / ? \\
\hline
\end{tabular}

SPOD à la forme négative

Le suffixe -iduwen se décompose dans l'ordre inattendu : -idu'PL', -w- 'NEG' et -en 'P3'.

Le faible nombre d'attestations ne permet pas de dire s'il existe une différence entre deux diathèses transitive et intransitive.

Il est possible mais douteux que la finale $-n$, aussi $-n n e$ soit une sorte d'anaphore. Cf. \$12.3. et \$13.3.

\section{Déictiques et autres pronoms}

13.1. Il existe plusieurs pronoms démonstratifs ou déictiques : anni 'ceci, celui-ci', andi 'cela, celui-là', akkiki ... agi 'l'un ... l'autre'. La forme alli, censée être attestée dans le Lion d'Urkeš, est fictive. 
D'autres formes pronominales incluent: $a b i$ 'qui ?', iya 'ce qui, celui qui', emeni, iyamani 'quiconque', šui, šummi 'tout'.

Ils se déclinent sensiblement comme mani 'il, elle P3SG' :

\begin{tabular}{|l|c|c|c|}
\hline & celui-ci & celui-là & P3SG \\
\hline Absolutif & anni & andi & mane \\
\hline Ergatif & annuiš & $?$ & manuš \\
\hline Base oblique & annu- & andu- & manu- \\
\hline \multicolumn{4}{|c}{ Déictiques (Singulier) }
\end{tabular}

\begin{tabular}{|l|c|c|c|}
\hline & ceux-ci & ceux-là & P3PL \\
\hline Absolutif & annil(la) & andil(la) & manel(la) \\
\hline Ergatif & $?$ & $?$ & manšuš \\
\hline Base oblique & $?$ & $?$ & manšu- \\
\hline
\end{tabular}

13.2. Emeni, iyamani 'quiconque' peut être suivi d'un verbe conjugué : Emeni purulli tapšelin Lubadagaš tapšuen! 'Quiconque voudra détruire le temple, que Nubadig le détruise !'.

13.3. Les PEM -anna 'P3SG' et -alla 'P3PL' servent aussi de pronoms anaphoriques pour le Singulier et le Pluriel. Ils peuvent raccourcis jusqu'à $-a$ ou - an pour 'P3SG' et -al pour 'P3PL'.

\section{Lexèmes invariables}

14.1. Il existe des adverbes primaires : adi 'ainsi', agabi 'de ce côté-ci', asabi 'de ce côté-là', henni 'maintenant', šatta 'ensemble', uya 'non'.

Il est possible de dériver des adverbes de manière à partir des adjectifs avec la marque de cas -ae 'INST'. Exemples : nirušae 'très vite', šennannae 'fraternellement'. Tiššan 'très' est sans doute lié à tei 'nombreux', par une sorte d'intensif : *te? $(u)^{\text {śann }}(a e)>$ tǐ̌šan.

14.2. Certains adverbiaux sont des noms positionnels POSI, à finale -u: telu 'en haut', tap̌š 'en bas', utburu 'sur le côté', kulu 'à nouveau'. Cf. \$9.14.

Le hourrite ne possède ni postpositions ni prépositions, mais certains noms communs, tels que abi 'tête', uruni 'derrière', etc., peuvent assûrer cette fonction. Cf. \$9.9. Le complément est au Datif et non au Génitif: Teššnppa abi 'devant Teššub' mais Tešš abi 'le visage de Teššub'.

14.3. D'autres mots invariables sont les conjonctions qui apparaissent en tête de phrase ou proposition et qui sont des organisateurs logiques du discours : ai- 'si', alase- 'est-ce que, si [style indirect]', anammi- 'ainsi, de cette façon', inna- 'quand', inu-, 
unu- 'combien, comment', panu- 'bien que', undu- 'alors, dès lors, au moment où'. A noter le cas de $u$ 'alors', qui semble emprunté à l'akkadien.

Il est fréquent que ces conjonctions soient suivies de PEM puis de conjonctions ENC. Anammidillan : anammi-dill-an 'Et ainsi nous'. Ces formes sont fréquentes dans la prose de la Lettre du Mittanni. A l'inverse le texte versifié de la Bilingue de Boğazköy ne favorise pas leur utilisation.

Il n'est pas certain que $u$ 'alors', empruntée à l'akkadien, puisse être suivie de PEM.

14.4. Une autre catégorie d'items invariables comprend les conjonctions enclitiques ENC, qui normalement sont suffixées au $1^{\text {er }}$ mot d'une phrase ou proposition. Leur contenu sémantique est faible. Exemples : -ma 'et puis', -an 'et [Ø]', -man 'mais', -nin '(?) en vérité'.

14.5. On aura noté qu'une finale - vn peut être une conjonction ENC - an, un PEM court anaphorique - an(na), un suffixe d'Optatif ou de Désidératif SPOD -en. Le phonème $n$ est impliqué dans un grand nombre de suffixes aux fonctions très différentes, ce qui peut entrainer une certaine confusion.

\section{Eléments de syntaxe}

15.1. Un nom peut être qualifié par un adjectif épithète, un complément du nom ou une proposition relative :

- ebri 'seigneur, maitre', un nom commun

- talawusi ebri 'gigantesque seigneur', avec un adjectif

- ebri Kummeniwi ‘seigneur de Kumme', avec un complément du nom au cas Génitif

Les éléments qualifiants peuvent être apposés au nom : Tě̌šub uru Kummeniwi talawušs ebri 'ô Teššub, gigantesque seigneur de la ville de Kumme' (KBo 32.15 Vo 4.14). Normalement les éléments régis prennent les mêmes marques de cas et articles que le nom qui les régit. Cf. $\$ 9.12$.

- arušan 'je l'ai donné(e)', arušauše 'que je [l']ai donné(e)'

- aruśauśeni nibarri 'la dot que j'ai donnée'

15.2. Les adjectifs peuvent être épithètes ou attributs du sujet. Dans ce dernier cas il est fréquent que leur terminaison soit $-a$. Cf. \$11.5. Par exemple Ibri-muša 'le seigneur est juste', un nom de personne similaire à l'akkadien Šarru-kinu.

Les raisons pour lesquelles certains adjectifs prennent un $-a$ ou ne le prennent pas ne sont pas claires. Cette finale est similaire à celle des verbes de mouvement. Cf. $\$ 10.1$. et $\$ 10.4$. 
Les adjectifs peuvent être utilisés adverbialement en suffixant la marque d'Instrumental -ae : nirušae 'très rapidement'.

15.3. Il n'y a pas de copule pour les adjectifs, contrairement aux noms, qui nécessitent man(n)-: erbi mannukku, manna tabi 'ce n'est pas un chien, c'est un homme' (adapté de KBo 32.14 I 7). Cf. $\$ 10.1$.

Dans les noms de personnes calqués sur le sémitique la copule est omise : Ebri-šäri 'Le seigneur est roi', Šärri-Teššn 'Teššub est roi'. La copule est parfois réduite à $-m a: E N-G A L=$ Ebri-Talmi 'le seigneur est grand' mais EN-LUGAL-ma = iwr-trm = Ebri-särrima 'le seigneur est roi'.

15.4. En général le noyau prédicatif d'une phrase est un verbe conjugué. Si le verbe est intransitif, le sujet est à l'Absolutif :

- Maneni šenniwwuwe pašsithi una 'Mane, [l']envoyé de mon frère vient'

Si le verbe est transitif, le sujet est au cas Ergatif, l'objet à l'Absolutif (\$9.9-10) et les suffixes verbaux SPAI sont ceux de la diathèse3 transitive ( $(12.5-6)$ :

- Tě̌šubaš pabanni ittiya 'Teššub fracasse la montagne'

L'ordre des mots est relativement libre puisque leur fonction est indiquée par des suffixes de cas, généralement non ambigus :

- sullubri erbiniš epheni talabhiya 'un pain šullubri le chien dans le four prend', (adapté de KBo 21.14 IV 9-10)

Certaines formes verbales archaïques transitives ressemblent à la diathèse1 au lieu de la diathèse 3 :

- nali pabanniš sidarna 'la montagne maudit le chevreuil'. Au lieu de šidarna, on attendrait plutôt šidar(n)iya

Un type particulier de phrases (passives) utilise des participes comme noyau prédicatif (Cf. \$11.7):

- pabanni amelanni tarriš 'que la montagne soit incendiée par le feu !', ici le cas Ergatif exprime le complément d'agent

15.5. Avec des verbes de parole, dire, parler, etc., il est fréquent que le sujet ne soit pas au cas Ergatif, que l'on attendrait :

- Megini tiwenna kadiya 'Megi dit ces mots', au lieu de *Meginiš

15.6. Un verbe peut qualifier un autre verbe en tant que Gérondif. Dans ce cas il prend le suffixe : -m-(a)-(e). Cf. \$10.12. La différence entre les formes les plus longues -mae et les variantes brèves n'est pas claire.

- tiwenna kunzimae kadiya 'il dit ces mots (étant) agenouillé'

Les voyelles intercalaires $\mathrm{a} / \mathrm{u} / \mathrm{i}$ sont normalement cohérentes avec les diathèses $1 / 2 / 3$. Kunzimae est logiquement une forme transitive qui signifie 'en pliant les genoux' 
15.7. Une proposition peut qualifier un nom. Dans ce cas la forme verbale conjuguée est suffixée par $-\check{s} e$, qui fait office de pronom relatif. Cf. $\$ 9.12, \$ 15.1$.

15.8. Le PEM anaphorique -(a)nna peut aussi servir à introduire des complétives :

- kadiyanna una 'il dit qu'il vient', litt. 'il le dit [que] il vient'

\section{Exemple de mise en œuvre}

KBo32.14 col. I lignes 1-15 : il s'agit d'un conte ou d'une fable, qui narre un chevreuil s'attirant des ennuis en proférant des malédictions irresponsables contre la montagne qui le nourrit. Il fait partie de la bilingue hourro-hittite découverte à Boğazköy en 1983 et 1985. Le sens général est une critique de l'ingratitude.

La tablette d'origine, publiée en copie autographe dans (KBo32 p.7), présente sensiblement cette apparence :

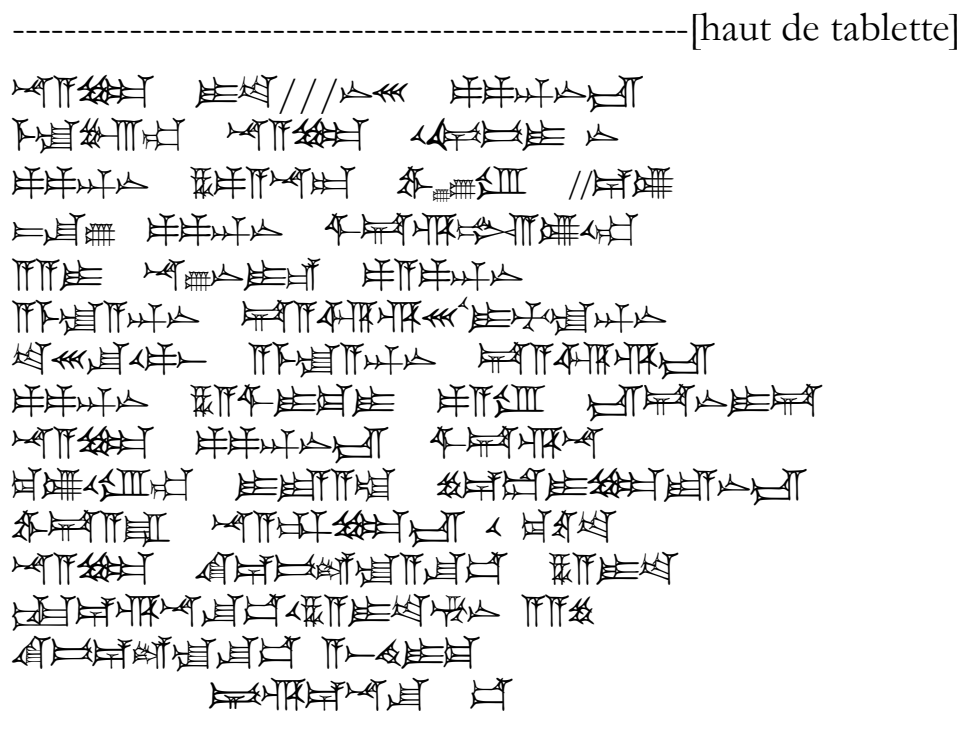

\section{Etape 1 : numéroter et translittérer}

A moins d'être à l'aise avec la lecture directe des signes cunéiformes, la $1^{\text {ère }}$ étape consiste à transformer les données en une TC standardisée. Les lignes ne sont pas numérotées sur les tablettes mais pour leur étude et leur référencement, il est utile de leur attribuer des numéros. On aboutit après ces ajouts à la situation suivante : 
[haut de tablette]

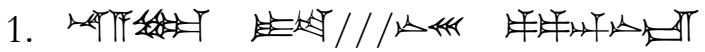

TC na-a-la i-te-////-na-eš pa-pa-an-na-iš

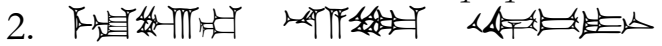

TC me-la-ab-um na-a-la u-ul-bi-i-na

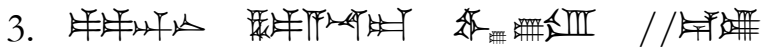

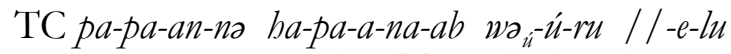



TC tab-su-ú pa-pa-an-na ši-ta-ro-il-lu-u-um

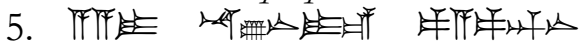

TC a-a-i na-ú-nə-i-e pa-a-pa-an-nə

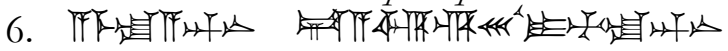

TC a-me-la-a-an-na ta-a-ar-ra-es "i-di-la-an-na

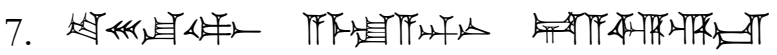

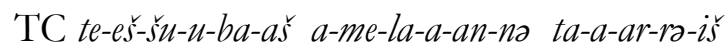

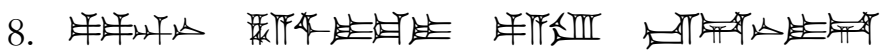

TC pa-pa-an-na ha-a-si-i-ma-i pa-a-ru iš-ta-na-i-ta

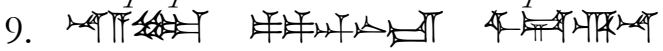

TC na-a-la pa-pa-an-na-iš ši-ta-ar-na

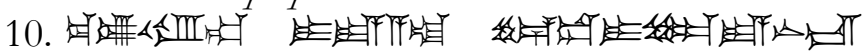

TC ku-lu-u-ru-um i-ia-a-at se-e-du-i-la-ia-no-iš

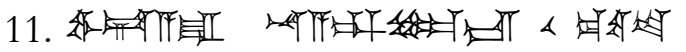

TC si-ta-a-ra na-a-al-bo-iš u ku-ut-te

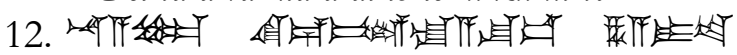

TC na-a-la gi-e-bi-il-la-a-šu-uš ba-a-i-te

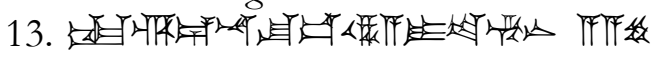

TC ka-re-e-na-šu-uš u ba-a-i-te-en-na a-a-še

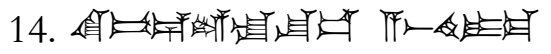

TC gi-bi-e-il-la-šsu-uš a-aš-bi-i-ma

15.

TC ga-re-e-na-su-us

Neu (StBo35, p. 74, 76) a également translittéré ce texte. Sa version diffère relativement aux points suivants :

- Neu harmonise les signes à lecture multiple, na et $l a$, avec le suivant, ou leur assigne d'office une valeur, $n i$ ou $l i$

- Ligne 2, il lit me-la-ah-bu-um mais le signe bu n'existe pas

- Lignes 6, 11, 13 il remplace le signe $4 u$ par $<:>$

- Ligne 2, il introduit avant na-a-la un $<:>$, qui n'existe pas

- Ligne 12, il lit ke au lieu de gi

- Ligne 13, il lit en 6 au lieu de en-na

Dans l'ensemble la version de Neu contient un certain nombre de principes interprétatifs, qui peuvent se discuter, mais aussi d'erreurs et d'omissions, qui sans aller jusqu'à fausser le texte ne lui sont pas fidèles. La tablette contient deux signes partiellement effacés : en Ligne1, sans doute [i] et en Ligne3 sans doute [te]. 
Vocabulaire :

ai 'si'

am- 'brûler, incendier'

aše 'chair, viande'

ašb- 'dépiauter, faire la peau'

edi 'corps'

ba- 'saisir, capturer'

haban- 'se rendre, se déplacer'

haš- 'entendre'

$i d$ - 'casser en morceaux'

ištani 'intérieur, milieu'

iya 'pourquoi'

kareni 'oiseleur'

kebili 'chasseur'

kut- 'tuer, abattre'

kul- 'dire', kulur- 'dire maintes fois'

melah- 'chasser, éloigner'

nali 'chevreuil, cerf'

nauni 'pâturage'

pabani 'montagne'

par- 'se mettre en colère'

šedu- 'engraisser'

sid- 'maudire', siidar- 'maudire à maintes reprises'

tapš́ 'en bas'

tari 'feu'

telu 'en haut'

$u$ 'et, et puis, alors'

ulbi 'autre'

wur- 'voir'

\section{Etape 2 : translittérer et normaliser}

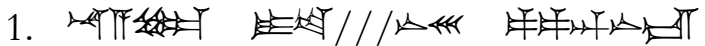

TC na-a-la i-te-///-nə-eš pa-pa-an-nə-iš

HCN nali edi rye]niš pabannis



TC me-la-ah-um na-a-la u-ul-bi-i-na

$\mathrm{HCN}$ melabum nali ulbini

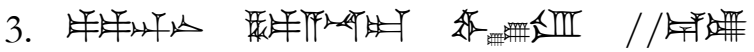

TC pa-pa-an-nә ba-pa-a-na-ab wә - iи-ru //-e-lu

HCN pabanni habanab wuru(b) [t]elu

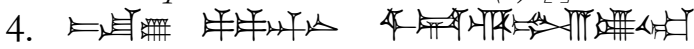

TC tab-šsu-ú pa-pa-an-na ši-ta-ro-il-lu-u-um

$\mathrm{HCN}$ tap̌̌u pabanni sidarillum

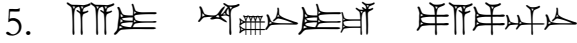

TC a-a-i na-ú-no-i-e pa-a-pa-an-nə

$\mathrm{HCN}$ ai nauniye pabanni 


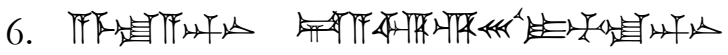
TC a-me-la-a-an-no ta-a-ar-ro-eš "i-di-la-an-no $\mathrm{HCN}$ amelanni tarriš u idelanni

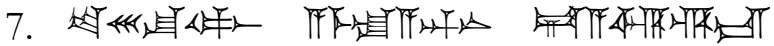
TC te-ers-šu-u-ba-aš a-me-la-a-an-no ta-a-ar-ro-ǐs HCN Tešsubaš amelanni tarriš

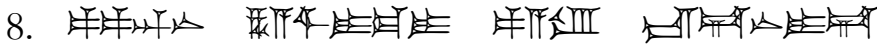

TC pa-pa-an-no ba-a-ši-i-ma-i pa-a-ru iš-ta-no-i-ta

$\mathrm{HCN}$ pabanni hašimae paru ištani(yi)da

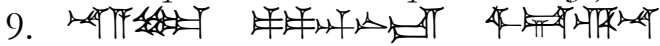

TC na-a-lo pa-pa-an-no-iš ši-ta-ar-na

$\mathrm{HCN}$ nali pabanniš sidarnale]

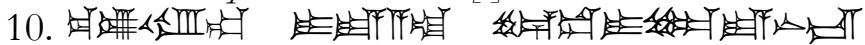

TC ku-lu-u-ru-um i-ia-a-at $\stackrel{s}{s}-e-d u-i-l o-i a-n o-i \dot{s}$

HCN kulurum iyat(ta) šeduiliyanis

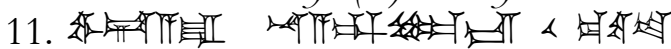



HCN sidara $[$ e 7 nallis u kutte

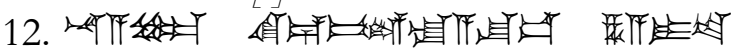

TC na-a-la gi-e-bi-il-la-a-šu-uš ba-a-i-te

HCN nali kebillašuš haide

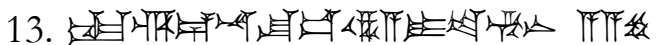

TC ka-re-e-na-šsu-uš u ha-a-i-te-en-no $a-a-s^{2} e$

$\mathrm{HCN}$ karen (n)ašus haidenni aše

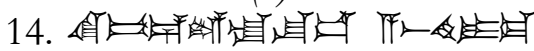

TC gi-bi-e-il-la- ${ }^{2} u-u s ̌ s$ a-ars-bi-i-ma

HCN kebillašuš aśhima(e)

15.



TC ga-re-e-na- ̌ru- $u \dot{s}$

$\mathrm{HCN}$ karen $(n) a \check{s} u \check{s}$

\section{Etape 3 : segmenter le texte en phrases}

La tablette sépare les mots avec des blancs mais on a affaire à un paragraphe complet sans ponctuation interne. Pour segmenter en propositions et phrases il faut s'appuyer sur différents indices, tels que les conjonctions, $u$ et $i y a$, les formes verbales, à finale $-m$ et $-b$, les marques de cas, en particulier l'ERG. On aboutit à la structure et ponctuation suivantes :

Nali edi[ye]nis pabanniš melabum,

nali ulbini pabanni habanab.

Wuru [b] [t]elu tapšsu pabanni sidarillum:

"Ai nauniye pabanni amelanni tarris

u idelanni Teššbaš, amelanni tarriš !»

Pabanni hašmae paru[b] istani(yi)da, nali pabanniš sidarna kulurum:

«Iyat(ta) šeduiliyaniš šidara nalliš? 
u kutte nali kebillašuš !

baide karen [n]ašuš !

haidenne aše kebillašušs!

ašbima[e] karen [n]ašuš!»

Analyse : Nali edi[ye]nišs pabanniš melabum

nali 'chevreuil' à l'ABs., objet du verbe melab-

edi[ye]nišs 'son corps' à l'ERG. DEF, épithète de pabanniš

pabanniš ‘la montagne' à l'ERG. DEF, sujet du verbe melab-

melahum 'chasser, expulser', au GER

Un cheureuil d'elle-même la montagne ayant chassé

Analyse : nali ulbini pabanni habanab

nali 'chevreuil' à l'ABS., sujet du verbe de mouvement haban-

ulbini 'l'autre' à l'ABS. DEF, épithète de pabanni

pabanni 'la montagne' à l'ABS. DEF, en fonction de LOC.

habanab 'se rendre, se déplacer', au PRET archaïque

[le dit] chevreuil en l'autre montagne se rendit

Analyse : Wuru[b] [t]elu tapšu pabanni šidarillum

wuru $[b]$ 'voir, regarder', sans finale $-b$ ou $-m$

telu 'en haut', nom positionnel à finale $-u$

tapš́ 'en bas', nom positionnel à finale $-u$

pabanni 'la montagne' à l'ABS. DEF, objet du verbe šidar-

šidarillum 'se mettre à maudire à maintes reprises', au GER

Il regarda de haut en bas, se mettant à maudire la montagne à maintes reprises :

Dans la traduction en hittite, le passage Wuru[b] [t]elu est rendu par 'il chercha querelle', mais ce n'est pas le sens littéral du texte hourrite. Il s'agit plutôt d'un commentaire qui donne le sens général, plutôt qu'une traduction. Si on interprête wuru $[\mathrm{m}]$ comme un GER, alors il faut rattacher cette phrase à la précédente et traduire regardant de haut en bas, commençant à la maudire à maintes reprises. En l'absence de marque formelle, les deux analyses sont possibles. Pabanni peut aussi être objet du verbe wur.

Analyse : «Ai nauniye pabanni amelanni tarriš

ai 'si', conjonction

nauniye 'son pâturage', à l'ABS. avec POss P3SG, sujet pabanni 'la montagne' à l'ABS. DEF, en fonction de LOC. amelanni 'incendié', PART au DESI, prédicat adjectival tarris' 'par le feu', à l'ERG. DEF, exprimant le moyen «S'il advient que [m]on pâturage en la montagne soit incendié par le feu

Le texte hourrite est en fait au style indirect.

Analyse : u idelanni Te šsubaš, amelanni tarriš !»

$u$ 'alors', conjonction

idelanni 'détruit, cassé', PART au DESI, prédicat adjectival Teššubaš par Teššub, à l'ERG. DEF, exprimant le moyen amelanni 'incendié', PART au DESI, prédicat adjectival tarrišc par le feu', à l'ERG. DEF, exprimant le moyen 
alors, je veux qu'elle soit cassée par Teššb [et] incendiée par le feu!»

Analyse : Pabanni hašimae paru[b] ištani(yi)da

pabanni 'la montagne' à l'ABS. DEF, sujet du verbe par-

hašimae 'en entendant [cela]', PART à l'INST.

paru $[b]$ 'être en colère', PRET sans finale $-b$ ou $-m$

istani(yi)da 'en son [for] intérieur', à l'ALL. L'ALL. exprime peutêtre l'acquisition par opposition au LOC. qui exprimerait l'état.

La montagne, en entendant [cela], se mit en colère en son [for] intérieur

Analyse : nali pabanniš sidarna kulurum

nali 'chevreuil' à l'ABS., objet du verbe šidarn-

pabanniš ‘la montagne' à l'ERG. DEF, sujet du verbe šidarn-

sidarna 'maudire'

kulurum 'dire maintes fois', au GER

Et la montagne de maudire maintes fois le chevreuil, en répétant:

L'interprétation de sidarna comme un nom au pluriel laisse nali sans fonction syntaxique, c'est pourquoi je préfère y voir un verbe, mais la finale $-a$ est étrange, on attendrait plutôt un PRET transitif du type $-i b$. Le même problème se pose dans la phrase suivante.

Analyse : «Iyat [ta] šeduiliyaniš šidara nalliš ?

iya 'pourquoi', conjonction

-t, PEM P1SG -(a)tta, objet du verbe TRA sidar-

šeduiliyaniš 'engraissé', PART à l'ERG. DEF, épithète de nali

sidara 'maudire'

nalliš'chevreuil' à l'ERG. DEF, sujet du verbe šidar-

Pourquoi le chevrenil, engraissé [par moi], me mandit-il?

La forme sidara, avec une finale - $a$ dans une forme conjuguée, semble exclure la diathèse transitive, mais la phrase contient un objet -atta et un sujet nallis. Une variante (archaïque ?) en - $a$ du PRES sidaria est une hypothèse possible.

Analyse : u kutte nali kebillašuš !

$u$ 'alors', conjonction

kutte 'abattre, tuer', verbe à l'OPTA (< HCN *kutt-eden)

nali 'chevreuil' à l'ABS., objet du verbe TRA kut-

kebillašuš 'les chasseurs', à l'ERG. DEF, sujet du verbe kut-

Alors, qu'ils abattent le chevreuil, les chasseurs!

Analyse : baide karen [n]ašuš !

baide 'saisir, capturer', verbe à l'OPTA (< $\mathrm{HCN} *$ ba-i-eden)

karen [n]ašuš 'les oiseleurs', à l'ERG. DEF, sujet du verbe $b a-$

qu'ils le capturent, les oiseleurs!

Analyse : haidenne ǎ́e kebillašuš !

baidenne 'saisir, capturer', verbe à l'OPTA ( $<\mathrm{HCN}$ * ba-i-eden) aše 'viande, chair' à l'ABS., objet du verbe $b a$ -

kebillašuš 'les chasseurs', à l'ERG. DEF, sujet du verbe kutqu'ils saississent sa chair, les chasseurs! 
Analyse : ašbima[e] karen [n]ašuš !»

ašbima[e] 'dépiauter', PART à l'INST.

karen [n]ašuš ‘les oiseleurs', à l'ERG. DEF, sujet du verbe ašben le dépiautant, les oiseleurs!»

\section{Etape 4 : traduction}

Nali edi[ye]nis pabanniš melabum,

Un chevrenil d'elle-même la montagne ayant chassé,

nali ulbini pabanni babanab.

[le dit] chevrenil en l'autre montagne se rendit.

Wuru[b] [t]elu tapšsu pabanni sidarillum:

Il regarda de haut en bas, se mettant à maudire la montagne à maintes reprises:

"Ai nauniye pabanni amelanni tarriš

« S'il advient que $[\mathrm{m}]$ on pâturage en la montagne soit incendié par le feu,

u idelanni Těšsubaš, amelanni tarriš !»

alors, je veux qu'elle soit cassée par Teššb [et] incendiée par le feu!»

Pabanni hašimae paru [b] ištani(yi)da,

La montagne, en entendant [cela], se mit en colère en son [for] intérieur,

nali pabanniš sidarn [i] a kulurum:

Et la montagne de maudire maintes fois le chevreuil, en répétant:

«Iyat [ta] šeduiliyaniš sidar [i] a nalliš?

Pourquoi le cheurenil, engraissé [par moi], me mandit-il?

u kutte nali kebillašuš!

Alors, qu'ils abattent le chevreuil, les chasseurs!

baide karen [n]ašuš !

qu'ils le capturent, les oiseleurs!

haidenni aše kebillašuš! !

qu'ils saississent sa chair, les chasseurs!

ašbima [e] karen[n]ašuš !»

en le dépiautant, les oiseleurs!» 
ABSTRACT - The paper aims at describing the main morphological, lexical and syntactical features of the Hurrian language, especially as it is attested in the documents of Mittannian or Anatolo-Hittite origin. It takes as its basis the language attested in the Mittanni Letter. This provides a state of the Hurrian language, which can be used as a universal reference for all actually attested forms. Issues pertaining to comparative endeavors, etymology, history, phonetics or writing systems are only dealt with when relevant. 